\title{
THE TENSOR PRODUCT OF EXCEPTIONAL REPRESENTATIONS ON THE GENERAL LINEAR GROUP
}

\author{
BY ANTHONY C. KABLE
}

ABSTRACT. - The exceptional representations are certain infinite-dimensional projective representations of the general linear group over a local field, somewhat analogous to the Weil representations of the symplectic group. We examine the decomposition of the tensor product of two exceptional representations. Our main results concern the multiplicity with which a given representation may occur in this product and the restrictions imposed upon a representation of the principal series by the assumption that it does occur.

(c) 2001 Éditions scientifiques et médicales Elsevier SAS

RÉSUMÉ. - Les représentations exceptionnelles sont certaines représentations projectives de dimension infinie du groupe linéaire général sur un corps local, apparentées aux représentations de Weil du groupe symplectique. Nous étudions la décomposition du produit tensoriel de deux représentations exceptionnelles. Nos résultats principaux concernent la multiplicité avec laquelle une représentation donnée apparaît dans ce produit, et les restrictions imposées à une représentation de la série principale par le fait qu'elle apparaît dans ce produit.

(C) 2001 Éditions scientifiques et médicales Elsevier SAS

\section{Introduction}

The exceptional representations are certain infinite-dimensional projective representations of the general linear group, GL $(r)$, over a local field. Equivalently, they may be viewed as linear representations of an appropriate double cover of GL $(r)$. The fundamental role played by the general linear group in many parts of mathematics makes its representations objects of wide interest. Beyond this, the exceptional representations attract attention for at least two reasons.

The first reason is analogical: on GL(2) the exceptional representations are identical with certain of the famous Weil representations of $\operatorname{GSp}(2)=\mathrm{GL}(2)$. While the higher Weil representations generalize these examples to the groups $\operatorname{GSp}(2 r)$, the higher exceptional representations generalize them to the groups $\mathrm{GL}(r)$. Thus the exceptional representations may be regarded as a GL $(r)$ analogue of the Weil representations and, given the remarkable properties possessed by the latter, seem worthy of close investigation.

The second reason is pragmatic: the exceptional representations play an essential role in the construction, by Bump and Ginzburg [5], of a Rankin-Selberg integral which represents the Langlands symmetric square $L$-function, $L\left(s, \Pi, \mathrm{sym}^{2}\right)$, of an automorphic representation, $\Pi$, of $\mathrm{GL}(r)$. To gain a more intimate understanding of this $L$-function would seem to require a detailed study of the ingredients in the construction, the exceptional representations prominent amongst them. Indeed, the work reported on here was carried out as part of a project, still underway, to investigate the structure of the local factors of $L\left(s, \Pi, \mathrm{sym}^{2}\right)$ at places where $\Pi$ is ramified. 
There is no succinct and unambiguous name for the double cover of $\operatorname{GL}(r)$ on which the exceptional representations have their natural home. The word "metaplectic", coined by Weil for a certain double cover of the symplectic group, has been pressed into service for covers of other classical groups also, thereby creating genuine ambiguity. Henceforth, bowing to convention, we shall use the generic term "metaplectic group" to refer to the group underlying our work.

The metaplectic group is itself a rather subtle object; its existence was not established in general until Matsumoto's remarkable 1969 paper [13]. Unlike Weil's metaplectic group, which was first constructed by giving a projective representation of $\operatorname{Sp}(2 r)$, the metaplectic group considered here was initially constructed group-theoretically. Even now, no a priori construction of the exceptional representations which would yield a natural proof of the existence of the metaplectic group is known. The subsequent history of the metaplectic group in the literature has been cloudy, with several erroneous statements finding their way into print. We shall use the work of Banks, Levy and Sepanski [1] as a convenient and reliable reference for the facts we require.

The exceptional representations of $\mathrm{GL}(r)$ were first introduced in generality by Kazhdan and Patterson in [12]. It is convenient to extend their definition somewhat to include representations of covers of products of several general linear groups and this is the first task in Section 5. Theorem 5.1 shows that, with this extended definition, the family of exceptional representations interacts smoothly with changing groups via parabolic induction and Jacquet functors and with the metaplectic tensor product. Next, in Theorems 5.2, 5.3 and 5.4, we compute the derivatives of the exceptional representations of $\mathrm{GL}(r)$. The second derivative has been considered previously by Bump and Ginzburg [5], who showed that it must again be exceptional but did not need to identify it precisely. It emerges that the first derivative is either an exceptional representation or a sum of such and that the higher derivatives are zero (subject, at present, to a technical hypothesis). As well as their applications to proving the main results of this paper, these facts are important in studying models of the exceptional representations akin to the Whittaker model. The author will return to this topic elsewhere.

Our main results, proved in Section 6, must appear rather technical at first sight and we certainly owe the reader some explanation of their context and the motivations for expecting them to be true. Simplifying the notation for the purposes of exposition, we let $\vartheta$ denote an exceptional representation of the metaplectic group. Our aim is to study the inner tensor product $\vartheta \otimes \vartheta$, where the two exceptional representations need not be the same. Since both exceptional representations live on the same cover of $\mathrm{GL}(r)$, the tensor product may be regarded as a linear representation of $\mathrm{GL}(r)$.

To see why this tensor product might be interesting, recall the role played by $\vartheta$ in the RankinSelberg integral for $L\left(s, \Pi, \mathrm{sym}^{2}\right)$. This integral is a product of local integrals over the places of the ground field and the integrand in each local integral is a product of a function coming from a model of the local factor of $\Pi$ and two functions which are associated with function space models of $\vartheta$. The representation-theoretic interpretation of a product of functions belonging to function space models for several representations is as a vector in the tensor product of the representations. Thus we are naturally led to consider the representation $\vartheta \otimes \vartheta \otimes \pi$, where $\pi$ is a local factor of $\Pi$. A more precise examination of the integral suggests, in fact, that this representation should carry a GL $(r)$-invariant linear functional (essentially the residue) when $L\left(s, \pi, \mathrm{sym}^{2}\right)$ has a pole at $s=s_{0}$. (The value of $s_{0}$ depends on a normalization; it is the rightmost location at which a pole is possible.)

The local Langlands conjectures predict the existence of a lift from $\mathrm{SO}(r)$ to $\mathrm{GL}(r)$, if $r$ is even, and from $\operatorname{Sp}(r-1)$ to $\mathrm{GL}(r)$, if $r$ is odd, associated with the natural inclusion of $\mathrm{SO}(r, \mathbb{C})$ into $\mathrm{GL}(r, \mathbb{C})$. It will be referred to here as the tautological lift. It is also predicted, at least for representations, $\pi$, of GL $(r)$ with trivial central character, that $\pi$ should lie in the image of the 
tautological lift if and only if $L\left(s, \pi, \mathrm{sym}^{2}\right)$ has a pole at $s=s_{0}$. Thus we are led to expect that there should be a connection between the existence of a GL $(r)$-invariant linear form on $\vartheta \otimes \vartheta \otimes \pi$ and the appearance of $\pi$ in the image of the tautological lift. The first precise formulation of such a conjecture is due to Savin and appears in [15].

The considerations of the last two paragraphs provide the motivation for the definition of a balanced character (Definition 6.2) and for guessing at the truth of something like Theorem 6.3. The idea is that a character of the maximal torus of $\operatorname{GL}(r)$ is balanced when it looks as if it arises from a character of the maximal torus of $\mathrm{SO}(r)$. The theorem says that, if $\pi$ is a principal series representation, then $\vartheta \otimes \vartheta \otimes \pi$ can carry an invariant linear functional only if the character corresponding to $\pi$ is balanced. The theorem should be able to be improved by replacing the condition $\chi_{j}^{2} \chi_{j^{*}}^{2}=1$ in the definition of a balanced character by $\chi_{j} \chi_{j^{*}}=1$. However, our current methods do not seem able to yield this refinement.

The majority of the other results in Section 6 are to the effect that the space of invariant linear functionals on $\vartheta \otimes \vartheta \otimes \pi$ is at most one-dimensional under various conditions. We can prove this unconditionally for irreducible $\pi$ on GL(2) and GL(3) (Theorem 6.5), for sufficiently general $\pi$ on any GL $(r)$ (Corollary 6.1) and for sufficiently general $\pi$ in the balanced part of the principal series (Theorem 6.4). The author conjectures that the space of invariant linear functionals is in fact always at most one-dimensional when $\pi$ is an irreducible representation. Given the analogy between the exceptional representations and the Weil representations, this may be compared to the final conjecture stated in [14]. In that paper the tensor product considered is with a nongenuine representation, but a moment's thought shows that the conjecture would imply the analogue for the Weil representations of our conjecture for the exceptional representations (and much more).

\section{The metaplectic group}

In this section we shall review those properties of the metaplectic group that will be required below. The notation introduced here will be used constantly in later sections.

Throughout, $F$ will be a non-archimedean local field, not of characteristic 2 . Given such a field there is a map $(\cdot, \cdot): F^{\times} \times F^{\times} \rightarrow \mu_{2}$, where $\mu_{2}=\{ \pm 1\}$, called the quadratic Hilbert symbol ([17], IX.5, p. 185ff). This symbol is symmetric and bimultiplicative and its left kernel is equal to the subgroup of squares in $F^{\times}$.

Let $\mathbb{P}$ be the set of all finite tuples of non-zero natural numbers and, for $\gamma=\left(r_{1}, \ldots, r_{k}\right) \in \mathbb{P}$, define the size of $\gamma$ to be $|\gamma|=r_{1}+\cdots+r_{k}$. An element $\gamma \in \mathbb{P}$ may be regarded as an ordered partition of $|\gamma|$. We partially order $\mathbb{P}$ by declaring that $\left(r_{1}, \ldots, r_{k}\right) \leqslant\left(s_{1}, \ldots, s_{\ell}\right)$ if and only if the partition $r_{1}+\cdots+r_{k}$ is a refinement of $s_{1}+\cdots+s_{\ell}$. Note that, if $\gamma, \delta \in \mathbb{P}$ are comparable under this order, then $|\gamma|=|\delta|$. We shall denote by $\gamma_{0}$ any of the minimal elements of $\mathbb{P}$ under this partial order; any such element has the form $(1, \ldots, 1)$. Whenever we discuss elements of $\mathbb{P}$, their size will be fixed throughout the discussion and so no confusion will arise from this ambiguous notation. Note that there is an obvious concatenation operation on $\mathbb{P}$; the concatenation of $\gamma_{1}$ and $\gamma_{2}$ will be written as $\left(\gamma_{1}, \gamma_{2}\right)$.

We denote by $G(r)$ the group $\operatorname{GL}(r, F)$. As usual, the root system of $G(r)$ may be identified with the set $\{(i, j) \mid 1 \leqslant i, j \leqslant r, i \neq j\}$ and, under this identification, $\Delta=\{(i, i+1) \mid$ $i=1, \ldots, r-1\}$ corresponds to the standard choice of positive simple system. For any $\gamma=$ $\left(r_{1}, \ldots, r_{k}\right) \in \mathbb{P}$ of size $r$ we let $\Delta(\gamma)$ be the complement in $\Delta$ of the set $\left\{\left(r_{1}, r_{1}+1\right), \ldots,\left(r_{1}+\right.\right.$ $\left.\left.\cdots+r_{k-1}, r_{1}+\cdots+r_{k-1}+1\right)\right\}$ and define $Q(\gamma) \leqslant G(r)$ to be the standard parabolic subgroup of $G(r)$ corresponding to $\Delta(\gamma)$ ([4], §14.17, p. 197). The group $G(\gamma)=G\left(r_{1}\right) \times \cdots \times G\left(r_{k}\right)$, regarded as embedded in the natural way as a block-diagonal subgroup of $G(r)$, is a Levi 
subgroup of $Q(\gamma)$. We let $N(\gamma)$ be the unipotent radical of $Q(\gamma)$ and, for $\gamma \leqslant \delta$, define $N(\delta, \gamma)=G(\delta) \cap N(\gamma)$. The Weyl group, $W$, of $G(r)$ is generated by the set $\left\{s_{\alpha} \mid \alpha \in \Delta\right\}$ of simple reflections. For $\gamma \in \mathbb{P}$ we let $W(\gamma)$ be the subgroup of $W$ generated by the set $\left\{s_{\alpha} \mid \alpha \in \Delta(\gamma)\right\}$. The group $W(\gamma)$ is naturally identified with the Weyl group of $G(\gamma)$.

The metaplectic group, as the term will be used here, is a central extension, $\widetilde{G}(r)$, of $G(r)$ by $\mu_{2}$. That is, it is a group which fits into a short exact sequence

$$
\{1\} \longrightarrow \mu_{2} \longrightarrow \widetilde{G}(r) \stackrel{p_{r}}{\longrightarrow} G(r) \longrightarrow\{1\},
$$

where $\mu_{2}$ injects into the center of $\widetilde{G}(r)$. For an account of the construction and principal properties of $\widetilde{G}(r)$ we refer to [1], which, in turn, draws upon the seminal work of Matsumoto in [13]. As we review the properties of the metaplectic group we shall make specific reference to results in [1] as appropriate.

For any subgroup, $H$, of $G(r)$ we let $\widetilde{H}=p_{r}^{-1}(H)$. This defines, in particular, a group $\widetilde{G}(\gamma)$ for any $\gamma=\left(r_{1}, \ldots, r_{k}\right) \in \mathbb{P}$. It is important to note that $\widetilde{G}(\gamma)$ is not isomorphic to the direct product of the $\widetilde{G}\left(r_{j}\right)$ with the copies of $\mu_{2}$ in each factor amalgamated, as one might initially expect. Rather, if $\gamma_{1}, \gamma_{2} \in \mathbb{P}, r_{1}=\left|\gamma_{1}\right|, r_{2}=\left|\gamma_{2}\right|, r=r_{1}+r_{2}$ and $g_{1}, g_{2} \in G\left(\gamma_{1}, \gamma_{2}\right)$ are such that $p_{r}\left(g_{1}\right) \in G\left(\gamma_{1}\right) \times\left\{I_{r_{2}}\right\}$ and $p_{r}\left(g_{2}\right) \in\left\{I_{r_{1}}\right\} \times G\left(\gamma_{2}\right)$ then

$$
g_{1} g_{2}=\left(\operatorname{det}\left(g_{1}\right), \operatorname{det}\left(g_{2}\right)\right) g_{2} g_{1},
$$

where det is being interpreted as a function on $\widetilde{G}(r)$ by composition with $p_{r}$. This formula follows from [1], §3, Theorem 11.

The sequence (2.1) corresponds in the usual way to a class in the cohomology group $\mathrm{H}^{2}\left(G(r), \mu_{2}\right)$, where $G(r)$ acts trivially on the coefficients, and choosing a cocycle to represent this class is equivalent to choosing a section $\mathrm{s}: G(r) \rightarrow \widetilde{G}(r)$ of the map $p_{r}$. We shall choose $\mathbf{s}$ in such a way that the resulting cocycle, $\sigma_{r}$, agrees with that constructed in [1], §3. If $h=\operatorname{diag}\left(h_{1}, \ldots, h_{r}\right)$ and $h^{\prime}=\operatorname{diag}\left(h_{1}^{\prime}, \ldots, h_{r}^{\prime}\right)$ are in $G\left(\gamma_{0}\right)$ then, by [1], $\S 3$, Theorem 7(a), we have

$$
\sigma_{r}\left(h, h^{\prime}\right)=\prod_{i<j}\left(h_{i}, h_{j}^{\prime}\right) .
$$

The restriction of $\mathbf{s}$ to $N\left(\gamma_{0}\right)$ is a homomorphism by [1], §3, Theorem 7(e) and we let $N^{*}(\gamma)=\mathbf{s}(N(\gamma))$ for any $\gamma \in \mathbb{P}$. Thus $N^{*}(\gamma)$ is a subgroup of $\widetilde{G}(r)$ isomorphic to $N(\gamma)$ and we have $\widetilde{Q}(\gamma)=\widetilde{G}(\gamma) \cdot N^{*}(\gamma)$ and $\widetilde{G}(\gamma) \cap N^{*}(\gamma)=\{e\}$. Furthermore, $N^{*}(\gamma)$ is a normal subgroup of $\widetilde{Q}(\gamma)$.

For $\gamma \in \mathbb{P}$ we define

$$
G^{2}(\gamma)=\left\{g \in G(\gamma) \mid \operatorname{det}(g) \in\left(F^{\times}\right)^{2}\right\}
$$

and

$$
G_{2}(\gamma)=\left\{\left(g_{1}, \ldots, g_{k}\right) \in G(\gamma) \mid \operatorname{det}\left(g_{j}\right) \in\left(F^{\times}\right)^{2}\right\} .
$$

Let $Z(\gamma)$ denote the center of $G(\gamma)$ and put $Z_{2}(\gamma)=Z(\gamma) \cap G_{2}\left(\gamma_{0}\right)$.

It will be convenient to have a formula for the commutator $[\tilde{g}, \tilde{z}]=\tilde{g} \tilde{z} \tilde{g}^{-1} \tilde{z}^{-1}$ when $\tilde{g} \in \widetilde{G}(r)$ and $\tilde{z} \in \widetilde{Z}(r)$. Using (2.3) it is easily found that

$$
[\tilde{g}, \tilde{z}]=(\operatorname{det}(g), \lambda)^{r-1},
$$


where $p_{r}(\tilde{z})=\lambda I_{r}$ and $p_{r}(\tilde{g})=g$. As a consequence, $Z(\widetilde{G}(r))$, the center of $\widetilde{G}(r)$, is given by

$$
Z(\widetilde{G}(r))= \begin{cases}\widetilde{Z}(r) & \text { if } r \text { is odd, } \\ \widetilde{Z}_{2}(r) & \text { if } r \text { is even. }\end{cases}
$$

It also follows from (2.6) that the group $\widetilde{Z}(r)$ is abelian regardless of the parity of $r$.

Let us give $G(r)$ the weakest topology under which all polynomial functions from $G(r)$ to $F$ are continuous. With this topology, $G(r)$ becomes an $\ell$-group, that is, a Hausdorff topological group which has a neighborhood base at the identity consisting of compact open subgroups. There is no notion of a polynomial function on $\widetilde{G}(r)$, but it is nevertheless possible to equip it with a topology under which it becomes an $\ell$-group and such that the map $p_{r}$ in (2.1) becomes a local homeomorphism. For a discussion of this see [11], §5. In future we shall always understand the metaplectic group to carry this topology.

\section{Metaplectic tensor products}

One of the basic constructions in the representation theory of $G(r)$ is to start with a list of representations, one for each of $G\left(r_{1}\right), \ldots, G\left(r_{k}\right)$, and then form their tensor product to obtain a representation of $G(\gamma), \gamma=\left(r_{1}, \ldots, r_{k}\right)$. Since $\widetilde{G}(\gamma)$ is not simply the amalgamated direct product of the various $\widetilde{G}\left(r_{j}\right)$, this construction cannot be carried over directly to the metaplectic case and our aim in this section is to find a replacement which shares as many of the useful properties of the tensor product as possible.

To be more precise we first require some notation and terminology. If, for $j=1, \ldots, k, H_{j}$ is a subgroup of $\widetilde{G}\left(r_{j}\right)$ containing $\mu_{2}$, then we define

$$
H_{1} \tilde{\times} H_{2} \widetilde{\times} \cdots \widetilde{\times} H_{k}=p_{r_{1}+\cdots+r_{k}}^{-1}\left(p_{r_{1}}\left(H_{1}\right) \times \cdots \times p_{r_{k}}\left(H_{k}\right)\right),
$$

where $p_{r_{1}}\left(H_{1}\right) \times \cdots \times p_{r_{k}}\left(H_{k}\right)$ is regarded as a subgroup of $G\left(r_{1}+\cdots+r_{k}\right)$ via the standard block-diagonal embedding of $G\left(r_{1}\right) \times \cdots \times G\left(r_{k}\right)$ into $G\left(r_{1}+\cdots+r_{k}\right)$. In particular, with $\gamma=\left(r_{1}, \ldots, r_{k}\right)$, we have $\widetilde{G}(\gamma)=\widetilde{G}\left(r_{1}\right) \tilde{\times} \cdots \widetilde{\times} \widetilde{G}\left(r_{k}\right)$.

We shall make use of the standard terminology of representation theory without explanation in what follows. For concepts specific to the representation theory of $\ell$-groups we shall follow the usage of [2]. Further, a representation of $\widetilde{G}(\gamma)$ will be called genuine if it is not trivial on $\mu_{2}$.

Given $\pi_{j}$ a representation of $\widetilde{G}\left(\gamma_{j}\right)$ for $j=1,2$, we aim to define a metaplectic tensor product $\pi_{1} \widetilde{\otimes} \pi_{2}$ which is to be a representation of $\widetilde{G}\left(\gamma_{1}\right) \widetilde{\times} \widetilde{G}\left(\gamma_{2}\right)$. In [10], Huang addressed this problem for the real metaplectic group in the case where $\pi_{1}$ and $\pi_{2}$ are irreducible and in [6] the problem is briefly discussed over a non-archimedean field, again for irreducible representations. This is too restrictive for our applications and we shall focus instead on genuine admissible representations of finite length.

If $\pi$ is a representation of a group $G$ with center $Z$ and $\omega$ is a character of $Z$ then we say that $\pi$ admits $\omega$ if there is a non-zero subquotient of $\pi$ on which $Z$ acts via $\omega$. If $G$ is an $\ell$-group and $\pi$ is an admissible indecomposable representation of $G$ of finite length then $\pi$ admits one and only one character of $Z$, which we shall denote by $\omega_{\pi}$.

The following lemma is standard with "irreducible" in place of "indecomposable" and the usual proof is easily modified to cover the more general case.

Lemma 3.1. - Let $G$ be a group, $H$ a normal subgroup of finite index and $\pi$ an indecomposable representation of $G$. Let $\sigma$ be any summand of $\left.\pi\right|_{H}$ and suppose that, if $g \notin H$, 
then $\operatorname{Hom}_{H}\left({ }^{g} \sigma, \sigma\right)=\{0\}$, where ${ }^{g} \sigma$ denotes the conjugate representation ${ }^{g} \sigma(h)=\sigma\left(g^{-1} h g\right)$. Then $\pi \cong \operatorname{ind}_{H}^{G}(\sigma)$. Conversely, if $\sigma$ is an indecomposable representation of $H$ of finite length which satisfies the above condition, then $\operatorname{ind}_{H}^{G}(\sigma)$ is indecomposable.

The group $\widetilde{G}^{2}(\gamma)$ (see (2.4)) is a normal subgroup of $\widetilde{G}(\gamma)$ and $\widetilde{G}(\gamma) / \widetilde{G}^{2}(\gamma) \cong F^{\times} /\left(F^{\times}\right)^{2}$. If $\pi$ is a representation of a subgroup of $\widetilde{G}(\gamma)$ containing $\widetilde{G}^{2}(\gamma)$ then we shall denote by $\pi^{2}$ the restriction of $\pi$ to $\widetilde{G}^{2}(\gamma)$. Our next task is to determine how $\pi^{2}$ decomposes when $\pi$ is an indecomposable representation of $\widetilde{G}(\gamma)$.

Suppose that $\rho$ is a finite-dimensional indecomposable representation of $\widetilde{Z}(r)$. Using the wellknown classification of finite-dimensional indecomposable representations of $G(1)$ we easily see that $\left.\rho\right|_{\widetilde{Z}_{2}(r)}$ is indecomposable and that a cyclic vector for $\rho$ remains cyclic for $\left.\rho\right|_{\widetilde{Z}_{2}(r)}$. Furthermore, if a finite-dimensional representation of $\widetilde{Z}(r)$ has a cyclic vector and admits only one character then it must be indecomposable.

Proposition 3.1. - Let $\gamma \in \mathbb{P}, r=|\gamma|$ and suppose that $r$ is odd. Let $\pi$ be an admissible indecomposable representation of $\widetilde{G}(\gamma)$ of finite length. Then $\pi^{2}$ is indecomposable and if $\chi \in\left(\widetilde{G}(\gamma) / \widetilde{G}^{2}(\gamma)\right)^{\wedge}$, the group of characters of $\widetilde{G}(\gamma) / \widetilde{G}^{2}(\gamma)$, and $\operatorname{Hom}_{\widetilde{G}(\gamma)}(\chi \otimes \pi, \pi) \neq\{0\}$, then $\chi=\chi_{0}$, the trivial character. Moreover, we have

$$
\operatorname{ind} \underset{\widetilde{G}^{2}(\gamma)}{\widetilde{G}(\gamma)}\left(\pi^{2}\right) \cong \bigoplus_{\chi \in\left(\widetilde{G}(\gamma) / \widetilde{G}^{2}(\gamma) \widehat{)}\right.} \chi \otimes \pi .
$$

Proof. - We know from (2.7) that $Z(\widetilde{G}(r))=\widetilde{Z}(r)$ when $r$ is odd and so

$$
\mathbf{s}\left(t I_{r}\right) \in Z(\widetilde{G}(r)) \cap \widetilde{G}(\gamma) \subseteq Z(\widetilde{G}(\gamma)) \text { for all } t \in F^{\times} .
$$

Since $\operatorname{det}\left(\mathbf{s}\left(t I_{r}\right)\right)=t^{r}$ and $r$ is odd, we may choose coset representatives for $\widetilde{G}(\gamma) / \widetilde{G}^{2}(\gamma)$ from $\widetilde{Z}(r)$. The restriction to $\widetilde{Z}(r)$ of the character of $Z(\widetilde{G}(\gamma))$ admitted by $\chi \otimes \pi$ is $\left.\left.\chi\right|_{\widetilde{Z}(r)} \cdot \omega_{\pi}\right|_{\widetilde{Z}(r)}$ and this equals $\left.\omega_{\pi}\right|_{\widetilde{Z}(r)}$ if and only if $\left.\chi\right|_{\widetilde{Z}(r)}$ is trivial. Combining these two observations proves the second claim.

To show that $\pi^{2}$ is indecomposable we shall in fact prove more: if $D$ is a $\widetilde{G}^{2}(\gamma)$-submodule of $E_{\pi}$ then $D$ is stable under $\widetilde{G}(\gamma)$. Suppose not. Then $D$ cannot be stable under $\pi(\widetilde{Z}(r))$ and so we may choose $\xi \in D$ such that $V=\operatorname{span}_{\mathbb{C}}\{\pi(z) \xi \mid z \in \widetilde{Z}(r)\}$ is not contained in $D$. We claim that $V$ is finite-dimensional. If $p$ is the length of $\pi$, then $\left(\pi(z)-\omega_{\pi}(z)\right)^{p} E_{\pi}=\{0\}$ for all $z \in \widetilde{Z}(r)$ and this implies that, for every $z \in \widetilde{Z}(r)$,

$$
\operatorname{span}_{\mathbb{C}}\left\{\pi\left(z^{c}\right) \mid c \in \mathbb{Z}\right\}=\operatorname{span}_{\mathbb{C}}\left\{\pi\left(z^{c}\right) \mid c=0, \ldots,(p-1)\right\},
$$

where the spans are taken in the algebra $\operatorname{End}_{\widetilde{G}(\gamma)}\left(E_{\pi}\right)$. Since $\pi$ is admissible there is some $f \geqslant 0$ such that $\pi(z) \xi= \pm \xi$ provided that $p_{r}(z) \in\left(1+\mathcal{P}_{F}^{f}\right) I_{r}$, where $\mathcal{P}_{F}$ denotes the maximal ideal in the ring of integers of $F$. The group $F^{\times} /\left(1+\mathcal{P}_{F}^{f}\right)$ is finitely-generated and if we choose $z_{1}, \ldots, z_{b} \in \widetilde{Z}(r)$ so that $\left\{p_{r}\left(z_{1}\right), \ldots, p_{r}\left(z_{b}\right)\right\}$ generates it, then

$$
V=\operatorname{span}_{\mathbb{C}}\left\{\pi\left(z_{1}^{c_{1}}\right) \pi\left(z_{2}^{c_{2}}\right) \cdots \pi\left(z_{b}^{c_{b}}\right) \xi \mid c_{i}=0, \ldots,(p-1)\right\}
$$

which is visibly finite-dimensional. Now $V$ has a cyclic vector, by its very definition, and admits only the character $\omega_{\pi}$. By the remarks made above on the representation theory of $\widetilde{Z}(r)$ it 
follows first that $V$ is indecomposable and secondly that $V=\operatorname{span}_{\mathbb{C}}\left\{\pi(z) \xi \mid z \in \widetilde{Z}_{2}(r)\right\}$. But $\widetilde{Z}_{2}(r) \subseteq \widetilde{G}^{2}(\gamma)$ and hence $V \subseteq D$, a contradiction. This proves the claim and with it the first statement of the proposition.

Finally, the isomorphism is well known, relying as it does simply on the fact that $\widetilde{G}(\gamma) / \widetilde{G}^{2}(\gamma)$ is abelian.

Proposition 3.2. - Let $\gamma \in \mathbb{P}, r=|\gamma|$ and suppose that $r$ is even. Let $\pi$ be a genuine admissible indecomposable representation of $\widetilde{G}(\gamma)$ of finite length. Suppose that $\sigma$ is any of the indecomposable summands of $\pi^{2}$. Then

$$
\pi \cong \operatorname{ind} \underset{\widetilde{G}^{2}(\gamma)}{\widetilde{G}(\gamma)}(\sigma)
$$

$\chi \otimes \pi \cong \pi$ for all $\chi \in\left(\widetilde{G}(\gamma) / \widetilde{G}^{2}(\gamma)\right)^{\Upsilon}$ and $\pi^{2} \cong \bigoplus_{g \in \widetilde{G}(\gamma) / \widetilde{G}^{2}(\gamma)}{ }^{g} \sigma$. Moreover, if $g \notin \widetilde{G}^{2}(\gamma)$, then $\operatorname{Hom}_{\widetilde{G}^{2}(\gamma)}\left({ }^{g} \sigma, \sigma\right)=\{0\}$.

Proof. - Since $r$ is even, $\widetilde{Z}(r) \subseteq \widetilde{G}^{2}(\gamma)$ and it follows from (2.6) that $\widetilde{Z}(r)$ is central in $\widetilde{G}^{2}(\gamma)$. Since $\sigma$ is an indecomposable admissible representation of $\widetilde{G}^{2}(\gamma)$ of finite length, it admits a unique character $\omega_{\sigma}$ of $\widetilde{Z}(r)$. Since $\pi$ is genuine, so is $\sigma$ and hence $\omega_{\sigma}$. If $g \in \widetilde{G}(\gamma)$, then for $t \in F^{\times}$, we have

$$
\omega_{g_{\sigma}}\left(\mathbf{s}\left(t I_{r}\right)\right)=\omega_{\sigma}\left(g^{-1} \mathbf{s}\left(t I_{r}\right) g\right)=(\operatorname{det}(g), t)^{r-1} \omega_{\sigma}\left(\mathbf{s}\left(t I_{r}\right)\right)
$$

on using (2.6). Thus if $\omega_{g_{\sigma}}=\omega_{\sigma}$, then $(\operatorname{det}(g), t)^{r-1}=1$ for all $t \in F^{\times}$, which implies that $\operatorname{det}(g) \in\left(F^{\times}\right)^{2}$. From this we conclude that, if $g \notin \widetilde{G}^{2}(\gamma)$, then $\operatorname{Hom}_{\widetilde{G}^{2}(\gamma)}\left({ }^{g} \sigma, \sigma\right)=\{0\}$ and consequently an appeal to Lemma 3.1 shows that

$$
\pi \cong \operatorname{ind} \underset{\widetilde{G}^{2}(\gamma)}{\widetilde{G}(\gamma)}(\sigma)
$$

We have thus established the first and last claims of the proposition. The second follows from the first by a purely formal argument and the third from the first by an appeal to the Mackey subgroup theorem.

The group $\widetilde{G}^{2}\left(\gamma_{1}\right) \widetilde{\times} \widetilde{G}^{2}\left(\gamma_{2}\right)$ is isomorphic to $\left(\widetilde{G}^{2}\left(\gamma_{1}\right) \times \widetilde{G}^{2}\left(\gamma_{2}\right)\right) / B$, where $B=\{(\epsilon, \epsilon) \mid$ $\left.\epsilon \in \mu_{2}\right\}$. If $\pi_{1}$ is a genuine representation of $\widetilde{G}\left(\gamma_{1}\right)$ and $\pi_{2}$ is a genuine representation of $\widetilde{G}\left(\gamma_{2}\right)$, then $B$ acts trivially on $\pi_{1}^{2} \otimes \pi_{2}^{2}$ and so this may be regarded as a genuine representation of $\widetilde{G}^{2}\left(\gamma_{1}\right) \widetilde{\times} \widetilde{G}^{2}\left(\gamma_{2}\right)$. If $\omega_{1}$ is a genuine character of $\widetilde{Z}_{2}(r)$ and $\omega_{2}$ is a genuine character of $\widetilde{Z}_{2}(s)$, then we obtain a genuine character $\omega_{1} \otimes \omega_{2}$ of $\widetilde{Z}_{2}(r) \widetilde{\times} \widetilde{Z}_{2}(s)$ by a similar construction. Given characters $\omega_{1}$ and $\omega_{2}$ of groups which contain $\widetilde{Z}_{2}(r)$ and $\widetilde{Z}_{2}(s)$, respectively, we shall allow ourselves to write $\omega_{1} \otimes \omega_{2}$ for the tensor product, in this sense, of their restrictions to $\widetilde{Z}_{2}(r)$ and $\widetilde{Z}_{2}(s)$. Note that $\widetilde{Z}_{2}\left(\left|\gamma_{1}\right|\right) \widetilde{\times} \widetilde{Z}_{2}\left(\left|\gamma_{2}\right|\right)$ acts on $\pi_{1}^{2} \otimes \pi_{2}^{2}$ via the character $\omega_{\pi_{1}} \otimes \omega_{\pi_{2}}$. These remarks may be extended to the group $\widetilde{G}\left(\gamma_{1}\right) \widetilde{\times} \widetilde{G}^{2}\left(\gamma_{2}\right)$ since it is isomorphic to $\left(\widetilde{G}\left(\gamma_{1}\right) \times \widetilde{G}^{2}\left(\gamma_{2}\right)\right) / B$.

THEOREM 3.1. - Let $\pi_{j}$ be a genuine admissible indecomposable representation of $\widetilde{G}\left(\gamma_{j}\right)$ of finite length for $j=1,2$, and put $r=\left|\gamma_{1}\right|+\left|\gamma_{2}\right|$. Suppose that $\omega$ is a character of $Z(\widetilde{G}(r))$ such that

$$
\left.\omega\right|_{\widetilde{Z}_{2}(r)}=\left.\left(\omega_{\pi_{1}} \otimes \omega_{\pi_{2}}\right)\right|_{\widetilde{Z}_{2}(r)} .
$$


Then the representation

$$
\Pi=\operatorname{ind} \underset{\widetilde{G}^{2}\left(\gamma_{1}\right) \times \widetilde{\times} \widetilde{G}^{2}\left(\gamma_{2}\right)}{\widetilde{\widetilde{a}}\left(\gamma_{1}\right)} \widetilde{\widetilde{a}} \widetilde{\gamma_{1}}\left(\pi_{1}^{2} \otimes \pi_{2}^{2}\right)
$$

has an indecomposable summand on which $Z(\widetilde{G}(r))$ acts by $\omega$. Any two indecomposable summands of $\Pi$ on which $Z(\widetilde{G}(r))$ acts by the same character are isomorphic. The restriction of an indecomposable summand of $\Pi$ to $\widetilde{G}^{2}\left(\gamma_{1}\right) \widetilde{\times} \widetilde{G}^{2}\left(\gamma_{2}\right)$ is isomorphic to the direct sum of $\left[F^{\times}:\left(F^{\times}\right)^{2}\right]$ copies of $\pi_{1}^{2} \otimes \pi_{2}^{2}$ if both $\left|\gamma_{1}\right|$ and $\left|\gamma_{2}\right|$ are odd and to $\pi_{1}^{2} \otimes \pi_{2}^{2}$ otherwise.

Proof. - If $G$ is a group, $H$ is a normal subgroup of finite index and $J$ is an intermediate group with $J / H$ abelian then it is easy to check that $\operatorname{ind}_{H}^{G}\left(\left.\rho\right|_{H}\right) \cong \bigoplus_{\chi \in(J / H)^{\wedge i n d}}{ }_{J}(\chi \otimes \rho)$ for any representation $\rho$ of $J$. If we take $G$ to be $\widetilde{G}\left(\gamma_{1}\right) \widetilde{\times} \widetilde{G}\left(\gamma_{2}\right), H$ to be $\widetilde{G}^{2}\left(\gamma_{1}\right) \widetilde{\times} \widetilde{G}^{2}\left(\gamma_{2}\right), J$ to be $\widetilde{G}\left(\gamma_{1}\right) \widetilde{\times} \widetilde{G}^{2}\left(\gamma_{2}\right)$ and $\rho=\pi_{1} \otimes \pi_{2}^{2}$ then the above hypotheses are satisfied and we obtain

$$
\Pi \cong \bigoplus_{\chi \in\left(\widetilde{G}\left(\gamma_{1}\right) / \widetilde{G}^{2}\left(\gamma_{1}\right)\right)^{\wedge}} \operatorname{ind}_{\widetilde{G}\left(\gamma_{1}\right)}^{\widetilde{\widetilde{C}}\left(\gamma_{1}\right) \widetilde{\times} \widetilde{G}\left(\gamma_{2}\right)}\left(\left(\chi \otimes \pi_{1}\right) \otimes \pi_{2}^{2}\right) .
$$

If $g_{2} \in \widetilde{G}\left(\gamma_{2}\right)$ then we use the symbol $\chi_{g_{2}}$ to denote the character of $\widetilde{G}\left(\gamma_{1}\right) / \widetilde{G}^{2}\left(\gamma_{1}\right)$ given by $\chi_{g_{2}}\left(g_{1}\right)=\left(\operatorname{det}\left(g_{1}\right), \operatorname{det}\left(g_{2}\right)\right)$. The non-degeneracy of the Hilbert symbol implies that every element of $\left(\widetilde{G}\left(\gamma_{1}\right) / \widetilde{G}^{2}\left(\gamma_{1}\right)\right)^{\wedge}$ arises in this way and, by (2.2), we have $g_{2}^{-1} g_{1} g_{2}=\chi_{g_{2}}\left(g_{1}\right) g_{1}$ for all $g_{1} \in \widetilde{G}\left(\gamma_{1}\right)$ and $g_{2} \in \widetilde{G}\left(\gamma_{2}\right)$. From this it follows that, if $\rho_{1}$ is a representation of $\widetilde{G}\left(\gamma_{1}\right)$ and $\rho_{2}$ is a representation of $\widetilde{G}^{2}\left(\gamma_{2}\right)$, then ${ }^{g_{1} g_{2}}\left(\rho_{1} \otimes \rho_{2}\right)=\left(\chi_{g_{2}} \otimes{ }^{g_{1}} \rho_{1}\right) \otimes{ }^{g_{2}} \rho_{2}$ for $g_{1} \in \widetilde{G}\left(\gamma_{1}\right)$ and $g_{2} \in \widetilde{G}\left(\gamma_{2}\right)$.

If $g_{2} \in \widetilde{G}\left(\gamma_{2}\right)$, then

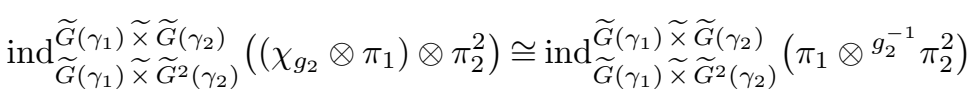

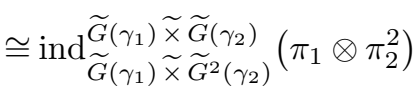

since $g_{2}^{-1} \pi_{2}^{2} \cong \pi_{2}^{2}$. Thus, if we put

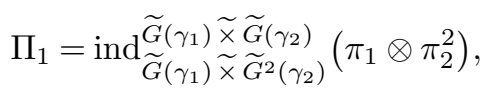

then $\Pi \cong \Pi_{1}^{\oplus\left[F^{\times}:\left(F^{\times}\right)^{2}\right]}$ and it follows that it is sufficient for us to prove our claims with $\Pi_{1}$ in place of $\Pi$.

If both $\left|\gamma_{1}\right|$ and $\left|\gamma_{2}\right|$ are odd, then $\pi_{2}^{2}$ is indecomposable by Proposition 3.1 and so $\pi_{1} \otimes \pi_{2}^{2}$ is indecomposable. If we take $g_{2} \in \widetilde{G}\left(\gamma_{2}\right) \backslash \widetilde{G}^{2}\left(\gamma_{2}\right)$, then

$$
\begin{aligned}
& \operatorname{Hom}_{\widetilde{G}\left(\gamma_{1}\right)} \widetilde{\times} \widetilde{G}^{2}\left(\gamma_{2}\right) \\
& \quad \cong \operatorname{Hom}_{\widetilde{G}\left(\gamma_{1}\right)}\left(\chi_{g_{2}} \otimes \pi_{1}, \pi_{1}\right) \otimes \operatorname{Hom}_{\widetilde{G}^{2}\left(\gamma_{2}\right)}\left(\pi_{2}^{2}, \pi_{2}^{2}\right)=\{0\},
\end{aligned}
$$

where we have used Proposition 3.1. Lemma 3.1 then implies that $\Pi_{1}$ is indecomposable. Since $r$ is even, (3.1) determines $\omega$ uniquely and it is equal to the character by which $Z(\widetilde{G}(r))$ acts on $\Pi_{1}$. The last claim follows from the Mackey subgroup theorem.

If either $\left|\gamma_{1}\right|$ or $\left|\gamma_{2}\right|$ is even, then we may assume without loss of generality that $\left|\gamma_{2}\right|$ is even (otherwise we would make the initial reduction step leading to $\Pi_{1}$ with the roles of $\gamma_{1}$ and $\gamma_{2}$ interchanged). Let $\sigma_{2}$ be an indecomposable summand of $\pi_{2}^{2}$. Then from Proposition 3.2 we have 
Let us put

$$
\begin{aligned}
\Pi_{1} & \cong \bigoplus_{g_{2} \in\left(\widetilde{G}\left(\gamma_{2}\right) / \widetilde{G}^{2}\left(\gamma_{2}\right)\right)} \operatorname{ind} \underset{\widetilde{G}\left(\gamma_{1}\right) \widetilde{\times}\left(\gamma^{2}\right) \widetilde{G}\left(\gamma_{2}\right)}{\widetilde{G}\left(\gamma_{2}\right)}\left(\pi_{1} \otimes{ }^{g_{2}} \sigma_{2}\right) \\
\cong & \bigoplus_{\chi \in\left(\widetilde{G}\left(\gamma_{1}\right) / \widetilde{G}^{2}\left(\gamma_{1}\right)\right)} \operatorname{ind} \underset{\widetilde{G}\left(\gamma_{1}\right)}{\widetilde{\times}\left(\gamma_{1}\right) \widetilde{G}\left(\gamma_{2}\right)}\left(\left(\chi \otimes \pi_{1}\right) \otimes \sigma_{2}\right)
\end{aligned}
$$

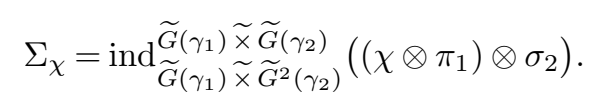

Since, by Proposition 3.2, all the conjugates of $\sigma_{2}$ are distinct, it follows as above that $\Sigma_{\chi}$ is indecomposable. If $\left|\gamma_{1}\right|$ is also even, then Proposition 3.2 implies that $\chi \otimes \pi_{1} \cong \pi_{1}$ for all $\chi \in\left(\widetilde{G}\left(\gamma_{1}\right) / \widetilde{G}^{2}\left(\gamma_{1}\right)\right)^{\wedge}$ and so $\Pi_{1} \cong \Sigma_{\chi_{0}}^{\oplus\left[F^{\times}:\left(F^{\times}\right)^{2}\right]}$. Again $r=\left|\gamma_{1}\right|+\left|\gamma_{2}\right|$ is even, (3.1) determines $\omega$ uniquely and it does equal the restriction of the central character of $\Sigma_{\chi_{0}}$ to $Z(\widetilde{G}(r))$. Computing the restriction of $\Sigma_{\chi_{0}}$ to $\widetilde{G}^{2}\left(\gamma_{1}\right) \widetilde{\times} \widetilde{G}^{2}\left(\gamma_{2}\right)$ in stages one finds that it equals $\pi_{1}^{2} \otimes \pi_{2}^{2}$ and the theorem is proved in this case.

Finally, suppose that $\left|\gamma_{2}\right|$ is even but that $\left|\gamma_{1}\right|$ is odd. Then $r=\left|\gamma_{1}\right|+\left|\gamma_{2}\right|$ is odd and so the group $Z(\widetilde{G}(r))=\widetilde{Z}(r)$ is contained in $\widetilde{G}\left(\gamma_{1}\right) \widetilde{\times} \widetilde{G}^{2}\left(\gamma_{2}\right)$ and is central in $\widetilde{G}\left(\gamma_{1}\right) \widetilde{\times} \widetilde{G}\left(\gamma_{2}\right)$. Thus $Z(\widetilde{G}(r))$ acts on $\Sigma_{\chi}$ via the restriction of the character $\left(\chi \otimes \omega_{\pi_{1}}\right) \otimes \omega_{\sigma_{2}}$ to $Z(\widetilde{G}(r))$. Since $\left|\gamma_{1}\right|$ is odd, this restriction determines $\chi$ and so the various $\Sigma_{\chi}$ are distinct. Moreover, as $\chi$ runs over $\left(\widetilde{G}\left(\gamma_{1}\right) / \widetilde{G}^{2}\left(\gamma_{1}\right)\right)^{\wedge}$, the restriction of $\left(\chi \otimes \omega_{\pi_{1}}\right) \otimes \omega_{\sigma_{2}}$ to $Z(\widetilde{G}(r))$ runs over all characters $\omega$ that satisfy (3.1). This proves the first two statements of the theorem in the final case and the last one follows as above.

DEFINITION 3.1. - Let $\pi_{j}$ be a genuine admissible indecomposable representation of $\widetilde{G}\left(\gamma_{j}\right)$ of finite length for $j=1,2$, and put $r=\left|\gamma_{1}\right|+\left|\gamma_{2}\right|$. Let $\omega$ be a character of $Z(\widetilde{G}(r))$ that satisfies

$$
\left.\omega\right|_{\widetilde{Z}_{2}(r)}=\left.\left(\omega_{\pi_{1}} \otimes \omega_{\pi_{2}}\right)\right|_{\widetilde{Z}_{2}(r)} .
$$

Then we shall denote by $\pi_{1} \widetilde{\otimes}_{\omega} \pi_{2}$ any indecomposable summand of the representation $\Pi$ defined in the statement of Theorem 3.1 on which $Z(\widetilde{G}(r))$ acts by the character $\omega$.

We require a few details about the construction of $\pi_{1} \widetilde{\otimes}_{\omega} \pi_{2}$ drawn from the proof of Theorem 3.1. To avoid referring directly to the proof in future, we isolate these in the following result.

COROLlaRY 3.1. - Let hypotheses and notation be as in Definition 3.1.

1. If $\left|\gamma_{1}\right|$ and $\left|\gamma_{2}\right|$ are both odd, then

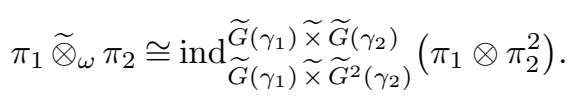

2. If $\left|\gamma_{1}\right|$ and $\left|\gamma_{2}\right|$ are both even, then

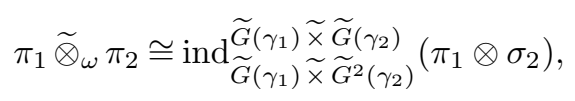

where $\sigma_{2}$ is any indecomposable summand of $\pi_{2}^{2}$.

3. If $\left|\gamma_{1}\right|$ is odd and $\left|\gamma_{2}\right|$ is even, then, for each $\omega$, there is a unique indecomposable summand $\sigma_{2}$ of $\pi_{2}^{2}$ such that

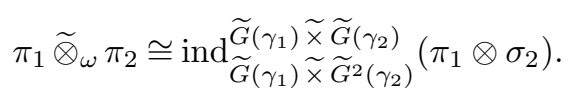


This summand is determined by requiring that the condition $\omega=\left.\left(\omega_{\pi_{1}} \otimes \omega_{\sigma_{2}}\right)\right|_{Z(\widetilde{G}(r))}$ hold. A similar result holds, mutatis mutandis, if $\left|\gamma_{1}\right|$ is even and $\left|\gamma_{2}\right|$ is odd.

Proof. - This follows immediately from Definition 3.1 and the proof of Theorem 3.1.

Next we shall state several propositions showing that the metaplectic tensor product $\widetilde{\otimes}$ shares enough of the useful properties of the usual tensor product to make it an effective substitute. We shall employ the notation introduced in Theorem 3.1 and Definition 3.1 throughout.

PROPOSITION 3.3. - Let $\pi$ be a genuine irreducible admissible representation of the group $\widetilde{G}\left(\gamma_{1}\right) \widetilde{\times} \widetilde{G}\left(\gamma_{2}\right)$ on which $Z(\widetilde{G}(r))$ acts by the character $\omega$. Then there are genuine irreducible admissible representations $\pi_{1}$ and $\pi_{2}$ of $\widetilde{G}\left(\gamma_{1}\right)$ and $\widetilde{G}\left(\gamma_{2}\right)$, respectively, such that $\pi \cong \pi_{1} \widetilde{\otimes}_{\omega} \pi_{2}$. Conversely, any such tensor product is irreducible.

Proof. - In what follows only the converse implication will be required and so the direct implication is left to the interested reader. We recall that restriction to and induction from a subgroup of finite index preserve both semisimplicity and the condition of being of finite length. Thus if $\pi_{1}$ and $\pi_{2}$ are irreducible, then $\pi_{1}^{2}$ and $\pi_{2}^{2}$ are semisimple, from which it follows that $\pi_{1}^{2} \otimes \pi_{2}^{2}$ is semisimple. Hence $\Pi$ as in Theorem 3.1 is semisimple. Since $\pi_{1} \widetilde{\otimes}_{\omega} \pi_{2} \leqslant \Pi$ is indecomposable, by construction, it follows that it is irreducible.

In the following proposition we use the notation $\widehat{\pi}$ for the contragredient of the representation $\pi$.

Proposition 3.4. - Suppose that $\pi_{j}$ is a genuine admissible representation of $\widetilde{G}\left(\gamma_{j}\right)$ of finite length for $j=1,2$, and that $\omega$ is a character of $Z(\widetilde{G}(r))$ such that $\pi_{1} \widetilde{\otimes}_{\omega} \pi_{2}$ is defined. Then $\widehat{\pi}_{1} \widetilde{\otimes}_{\omega^{-1}} \widehat{\pi}_{2}$ is defined and isomorphic to the contragredient of $\pi_{1} \widetilde{\otimes}_{\omega} \pi_{2}$.

Proof. - This follows from the fact that the operations of inducing from a subgroup of finite index and forming the contragredient commute.

PROPOSITION 3.5. - Suppose that $\pi_{j}$ is a genuine admissible indecomposable representation of $\widetilde{G}\left(\gamma_{j}\right)$ of finite length for $j=1,2,3$, and put $s=\left|\gamma_{1}\right|+\left|\gamma_{2}\right|+\left|\gamma_{3}\right|$. Then, for any character $\omega$ of $Z(\widetilde{G}(s))$ such that $\left.\omega\right|_{\widetilde{Z}_{2}(s)}=\left.\left(\omega_{\pi_{1}} \otimes \omega_{\pi_{2}} \otimes \omega_{\pi_{3}}\right)\right|_{\widetilde{Z}_{2}(s)}$, we have

$$
\pi_{1} \widetilde{\otimes}_{\omega}\left(\pi_{2} \widetilde{\otimes} \pi_{3}\right) \cong\left(\pi_{1} \widetilde{\otimes} \pi_{2}\right) \widetilde{\otimes}_{\omega} \pi_{3} .
$$

Here the two parenthetical metaplectic tensor products may be formed using any suitable character of $Z\left(\widetilde{G}\left(\left|\gamma_{2}\right|+\left|\gamma_{3}\right|\right)\right)$ and $Z\left(\widetilde{G}\left(\left|\gamma_{1}\right|+\left|\gamma_{2}\right|\right)\right)$, respectively.

Proof. - Using the definition of $\widetilde{\otimes}_{\omega}$ and the transitivity of induction it is routine to check that both $\pi_{1} \widetilde{\otimes}_{\omega}\left(\pi_{2} \widetilde{\otimes} \pi_{3}\right)$ and $\left(\pi_{1} \widetilde{\otimes} \pi_{2}\right) \widetilde{\otimes}_{\omega} \pi_{3}$ are isomorphic to any of the indecomposable summands of

$$
\operatorname{ind} \underset{\widetilde{G}^{2}\left(\gamma_{1}\right) \widetilde{\times}\left(\widetilde{G}^{2}\left(\gamma_{2}\right) \widetilde{\times} \widetilde{G}^{2}\left(\gamma_{3}\right)\right.}{\widetilde{G}} \widetilde{\widetilde{G}}\left(\pi_{1}^{2} \otimes \pi_{2}^{2} \otimes \pi_{3}^{2}\right)
$$

on which $Z(\widetilde{G}(s))$ acts by the character $\omega$.

Finally, we want to extend the definition of $\widetilde{\otimes}_{\omega}$ to allow factors which are not necessarily indecomposable. We shall call a representation $\pi$ of $\widetilde{G}(\gamma)$ homogeneous if it admits only one character of $Z(\widetilde{G}(\gamma))$. Thus indecomposable representations of finite length are homogeneous and if $\pi$ is a homogeneous representation, then it still makes sense to write $\omega_{\pi}$. If $\pi_{j}$ is a genuine admissible homogeneous representation of $\widetilde{G}\left(\gamma_{j}\right)$ of finite length for $j=1,2$, and $\omega$ 
is a character of $Z(\widetilde{G}(r))$ satisfying $\left.\omega\right|_{\widetilde{Z}_{2}(r)}=\left.\left(\omega_{\pi_{1}} \otimes \omega_{\pi_{2}}\right)\right|_{\widetilde{Z}_{2}(r)}$, then we define $\pi_{1} \widetilde{\otimes}_{\omega} \pi_{2}$ by requiring that $\widetilde{\otimes}_{\omega}$ distribute over direct sums. It follows from the Krull-Schmidt theorem in this category that this extension is well defined. The properties of $\widetilde{\otimes}_{\omega}$ given in Propositions 3.4 and 3.5 remain valid in the extended setting.

\section{A parade of functors}

The goal of this section is to introduce a number of functors whose properties will be essential in the proofs of our main results. Although we shall complete the theory in a number of points, most of what we say constitutes a review of known facts and so we shall be brief.

If $G$ is any $\ell$-group then we let $\mathcal{R}_{\text {alg }}(G)$ denote the category of algebraic representations of $G$. Suppose that $\gamma, \delta \in \mathbb{P}$ and that $\gamma \leqslant \delta$. Then we let $i_{\gamma, \delta}: \mathcal{R}_{\text {alg }}(\widetilde{G}(\gamma)) \rightarrow \mathcal{R}_{\text {alg }}(\widetilde{G}(\delta))$ be the normalized induction functor and $\varphi_{\delta, \gamma}: \mathcal{R}_{\text {alg }}(\widetilde{G}(\delta)) \rightarrow \mathcal{R}_{\text {alg }}(\widetilde{G}(\gamma))$ the normalized Jacquet functor corresponding to the groups $\widetilde{G}(\delta), \widetilde{G}(\gamma)$ and $N^{*}(\delta, \gamma)$ and the trivial character on $N^{*}(\delta, \gamma)$. The definitions of $i_{\gamma, \delta}$ and $\varphi_{\delta, \gamma}$ together with many properties enjoyed by these functors are given in [3], beginning on p. 444.

Let $\gamma, \delta \in \mathbb{P}$ with $|\gamma|=|\delta|=r$ and $\gamma \leqslant \delta$. We define the map $\mu_{\delta, \gamma}: \widetilde{G}(\gamma) \rightarrow \mathbb{R}_{+}^{\times}$to be the modular character of $\widetilde{G}(\gamma)$ acting by conjugation on $N^{*}(\delta, \gamma)$. It is the same as the modular character of $\widetilde{G}(\gamma)$ acting on $\widetilde{N}(\delta, \gamma)$ and, after composition with $p_{r}$, it also equals the modular character of $G(\gamma)$ acting on $N(\delta, \gamma)$. If $\beta$ is a third partition of $r$ and $\beta \leqslant \gamma \leqslant \delta$, then we have $N(\delta, \beta)=N(\delta, \gamma) \cdot N(\gamma, \beta)$ and $N(\delta, \gamma) \cap N(\gamma, \beta)=\{1\}$. It follows from this that $\mu_{\delta, \beta}=\mu_{\delta, \gamma} \cdot \mu_{\gamma, \beta}$ as characters of either $G(\beta)$ or of $\widetilde{G}(\beta)$.

The following result is a further example of the good behavior of the metaplectic tensor product defined in Section 3.

PROPOSITION 4.1. - Let $\pi_{j}$ be a genuine admissible homogeneous representation of $\widetilde{G}\left(\delta_{j}\right)$ of finite length for $j=1,2$, and suppose that $\gamma_{1} \leqslant \delta_{1}$ and $\gamma_{2} \leqslant \delta_{2}$. Then

$$
\varphi_{\left(\delta_{1}, \delta_{2}\right),\left(\gamma_{1}, \gamma_{2}\right)}\left(\pi_{1} \widetilde{\otimes}_{\omega} \pi_{2}\right) \cong \varphi_{\delta_{1}, \gamma_{1}}\left(\pi_{1}\right) \widetilde{\otimes}_{\omega} \varphi_{\delta_{2}, \gamma_{2}}\left(\pi_{2}\right)
$$

for every character $\omega$ of $Z\left(\widetilde{G}\left(\left|\delta_{1}\right|+\left|\delta_{2}\right|\right)\right)$ for which $\pi_{1} \widetilde{\otimes}_{\omega} \pi_{2}$ is defined.

Proof. - Set $\delta=\left(\delta_{1}, \delta_{2}\right)$ and $\gamma=\left(\gamma_{1}, \gamma_{2}\right)$. We begin by applying Theorem 5.2 of [3] to compute the composition of $\varphi_{\delta, \gamma}$ with induction from $\widetilde{G}\left(\delta_{1}\right) \widetilde{\times} \widetilde{G}^{2}\left(\delta_{2}\right)$ to $\widetilde{G}(\delta)$. Since we may choose coset representatives for $\widetilde{G}^{2}\left(\delta_{2}\right) \backslash \widetilde{G}\left(\delta_{2}\right)$ from $\widetilde{G}\left(\gamma_{2}\right)$, there is a single $\left(\widetilde{G}\left(\delta_{1}\right) \widetilde{\times} \widetilde{G}^{2}\left(\delta_{2}\right), \widetilde{G}(\gamma) \cdot N^{*}(\delta, \gamma)\right)$ double coset in $\widetilde{G}(\delta)$. Using this observation, we obtain an isomorphism of functors

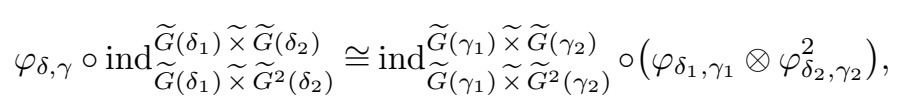

where $\varphi_{\delta_{2}, \gamma_{2}}^{2}: \mathcal{R}_{\text {alg }}\left(\widetilde{G}^{2}\left(\delta_{2}\right)\right) \rightarrow \mathcal{R}_{\text {alg }}\left(\widetilde{G}^{2}\left(\gamma_{2}\right)\right)$ is the Jacquet functor with respect to the trivial character on $N^{*}\left(\delta_{2}, \gamma_{2}\right)$. Also, $\left.\left.\right|_{\widetilde{G}^{2}\left(\gamma_{2}\right)} \circ \varphi_{\delta_{2}, \gamma_{2}} \cong \varphi_{\delta_{2}, \gamma_{2}}^{2} \circ\right|_{\widetilde{G}^{2}\left(\delta_{2}\right)}$, where the vertical bars represent restriction functors. If both $\left|\delta_{1}\right|=\left|\gamma_{1}\right|$ and $\left|\delta_{2}\right|=\left|\gamma_{2}\right|$ are odd, then it follows from Corollary 3.1 that

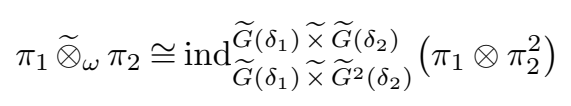


and similarly for the representations of $\widetilde{G}\left(\gamma_{1}\right)$ and $\widetilde{G}\left(\gamma_{2}\right)$. In this case the Proposition follows directly from (4.1).

If either $\left|\delta_{1}\right|$ or $\left|\delta_{2}\right|$ is even, then we shall assume that $\left|\delta_{2}\right|$ is even, the other case being similar. We may also assume that $\pi_{1}$ and $\pi_{2}$ are both indecomposable, since both sides of the proposed isomorphism respect direct sums. It follows from Corollary 3.1 that, for each possible character $\omega$, there is an indecomposable summand $\sigma_{2}$ of $\pi_{2}^{2}$ such that

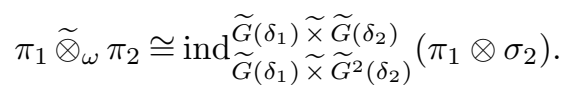

With this choice of $\sigma_{2}$ we have

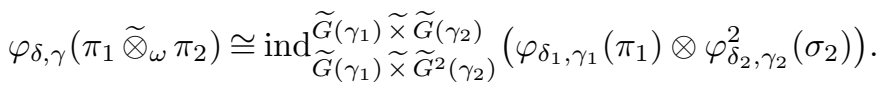

The representation $\varphi_{\delta_{2}, \gamma_{2}}\left(\pi_{2}\right)$ will generally have several indecomposable summands, say $\varphi_{\delta_{2}, \gamma_{2}}\left(\pi_{2}\right)=\rho_{1} \oplus \cdots \oplus \rho_{k}$. The indecomposable summands of $\rho_{j}^{2}$ are distinguished from one another by the character via which $\widetilde{Z}\left(\left|\gamma_{2}\right|\right)$ acts on them. We fix a particular character among the possibilities and let $\nu_{j}$ be the indecomposable summand of $\rho_{j}^{2}$ corresponding to that character for $j=1, \ldots, k$. It then follows from Proposition 3.2 that $\rho_{j}^{2} \cong \bigoplus_{g \in \widetilde{G}\left(\gamma_{2}\right) / \widetilde{G}^{2}\left(\gamma_{2}\right)}{ }^{g} \nu_{j}$ and so if we set $\nu=\nu_{1} \oplus \cdots \oplus \nu_{k}$, then

$$
\varphi_{\delta_{2}, \gamma_{2}}^{2}\left(\pi_{2}^{2}\right) \cong \bigoplus_{g \in \widetilde{G}\left(\gamma_{2}\right) / \widetilde{G}^{2}\left(\gamma_{2}\right)}{ }^{g} \nu
$$

and $\widetilde{Z}\left(\left|\gamma_{2}\right|\right)$ acts on each ${ }^{g} \nu$ via a single character. If the character via which $\widetilde{Z}\left(\left|\gamma_{2}\right|\right)$ acts on $\nu$ was chosen correctly, then it follows from (4.3) and Corollary 3.1 that

$$
\varphi_{\delta_{1}, \gamma_{1}}\left(\pi_{1}\right) \widetilde{\otimes}_{\omega} \varphi_{\delta_{2}, \gamma_{2}}\left(\pi_{2}\right) \cong \operatorname{ind} \underset{\widetilde{G}\left(\gamma_{1}\right)}{\widetilde{\widetilde{x}}\left(\gamma_{1}\right) \widetilde{\times} \widetilde{G}\left(\gamma_{2}\right)}\left(\varphi_{\delta_{1}, \gamma_{1}}\left(\pi_{1}\right) \otimes \nu\right) .
$$

In addition, $\pi_{2}^{2} \cong \bigoplus_{g \in \widetilde{G}\left(\gamma_{2}\right) / \widetilde{G}^{2}\left(\gamma_{2}\right)}{ }^{g} \sigma_{2}$, since we can choose coset representatives for $\widetilde{G}\left(\delta_{2}\right) / \widetilde{G}^{2}\left(\delta_{2}\right)$ from $\widetilde{G}\left(\gamma_{2}\right)$, and so

$$
\varphi_{\delta_{2}, \gamma_{2}}^{2}\left(\pi_{2}^{2}\right) \cong \bigoplus_{g \in \widetilde{G}\left(\gamma_{2}\right) / \widetilde{G}^{2}\left(\gamma_{2}\right)} g \varphi_{\delta_{2}, \gamma_{2}}^{2}\left(\sigma_{2}\right) .
$$

Since $\sigma_{2}$ is indecomposable, it is homogeneous for $\widetilde{Z}\left(\left|\delta_{2}\right|\right)$ and hence $\varphi_{\delta_{2}, \gamma_{2}}^{2}\left(\sigma_{2}\right)$ is homogeneous for $\widetilde{Z}\left(\left|\gamma_{2}\right|\right)$ and this group acts on each conjugate in (4.5) via a different character. Comparing homogeneous summands in (4.3) and (4.5) we see that there is some $g \in \widetilde{G}\left(\gamma_{2}\right)$ such that $\varphi_{\delta_{2}, \gamma_{2}}^{2}\left(\sigma_{2}\right) \cong{ }^{g} \nu$. Using this in (4.2) we obtain

$$
\varphi_{\delta, \gamma}\left(\pi_{1} \widetilde{\otimes}_{\omega} \pi_{2}\right) \cong \operatorname{ind} \underset{\widetilde{G}\left(\gamma_{1}\right)}{\widetilde{\widetilde{x}}\left(\gamma_{1}\right) \widetilde{\times} \widetilde{G}\left(\gamma_{2}\right)}\left(\varphi_{\delta_{1}, \gamma_{1}}\left(\pi_{1}\right) \otimes{ }^{g} \nu\right)
$$

If $\left|\gamma_{1}\right|$ is even then (4.4) and (4.6) imply at once that $\varphi_{\delta, \gamma}\left(\pi_{1} \widetilde{\otimes}_{\omega} \pi_{2}\right) \cong \varphi_{\delta_{1}, \gamma_{1}}\left(\pi_{1}\right) \widetilde{\otimes}_{\omega} \varphi_{\delta_{2}, \gamma_{2}}\left(\pi_{2}\right)$. If, on the other hand, $\left|\gamma_{1}\right|$ is odd, then the fact that $Z\left(\widetilde{G}\left(\left|\delta_{1}\right|+\left|\delta_{2}\right|\right)\right)$ acts via $\omega$ on the lefthand side of both (4.4) and (4.6) implies that $g \in \widetilde{G}^{2}\left(\gamma_{2}\right)$ and this, in turn, gives the required isomorphism. 
Our next task is to discuss the $\Phi$ and $\Psi$ functors introduced by Bernstein and Zelevinsky in connection with their theory of derivatives. This theory is described in its fully elaborated form in [3] after having been prefigured in [2] and [9]. At its heart is the subgroup $P(r)$ of $G(r)$ defined to be the stabilizer in $G(r)$ of the vector $(0,0, \ldots, 0,1) \in F^{r}$ when $G(r)$ acts on $F^{r}$ on the right in the usual way. The group $P(r)$ is called the mirabolic subgroup of $G(r)$. If $s>r$, then $P(r)$ may be regarded as a subgroup of $G(s)$ via the standard embedding $G(r) \hookrightarrow G(s)$. We define $Y_{r}=N((r),(r-1,1)) ; Y_{r}$ is equal to the unipotent radical of $P(r)$. The image of $Y_{r}$ under the homomorphism $\mathbf{s}: N\left(\gamma_{0}\right) \rightarrow N^{*}\left(\gamma_{0}\right)$ will be denoted by $Y_{r}^{*}$.

Let us fix a non-trivial continuous additive character $\psi$ of the field $F$. This gives rise to a character of $N\left(\gamma_{0}\right)$, also denoted by $\psi$, whose value at the matrix $\left(n_{a b}\right)$ is $\psi\left(n_{12}+\cdots+n_{r-1, r}\right)$. Since s : $N\left(\gamma_{0}\right) \rightarrow N^{*}\left(\gamma_{0}\right)$ is an isomorphism, $\psi$ may also be regarded as a character of $N^{*}\left(\gamma_{0}\right)$.

We now introduce six functors as shown in the diagram.

$$
\mathcal{R}_{\text {alg }}\left(\widetilde{G}(r-1) \stackrel{\frac{\Psi^{-}, \widehat{\Psi}^{-}}{\Psi^{+}}}{\longleftrightarrow} \mathcal{R}_{\text {alg }}(\widetilde{P}(r)) \stackrel{\Phi^{+}, \widehat{\Phi}^{+}}{\stackrel{\mathcal{R}_{\text {alg }}}{\stackrel{\Phi^{-}}{\longrightarrow}}}(\widetilde{P}(r-1)) .\right.
$$

Except for $\widehat{\Psi}^{-}$, which Bernstein and Zelevinsky do not discuss, all these functors are the exact analogues in the metaplectic setting of the eponomous functors described in [3]. It is important to note that both the Jacquet functors and the extension functors are normalized by a suitable modular character. The functor $\widehat{\Psi}^{-}$is defined as follows: if $\tau \in \mathcal{R}_{\text {alg }}(\widetilde{P}(r))$, then $\widetilde{G}(r-1)$ preserves the space of $Y_{r}^{*}$-invariant vectors in the space of $\tau$ and restricting $\tau$ to this subspace yields an algebraic representation of $\widetilde{G}(r-1)$. This representation twisted by the inverse squareroot of the modular character of $\widetilde{G}(r-1)$ acting on $Y_{r}^{*}$ will be, by definition, $\widehat{\Psi}^{-}(\tau)$. If $T \in \operatorname{Hom}_{\widetilde{P}(r)}\left(\tau_{1}, \tau_{2}\right)$, then $\widehat{\Psi}^{-}(T)$ will simply be the restriction of $T$ to the space of $\widehat{\Psi}^{-}\left(\tau_{1}\right)$. We note that $\widehat{\Psi}^{-} \Psi^{+}=\mathrm{Id}$.

The basic properties of $\Psi^{ \pm}, \Phi^{ \pm}$and $\widehat{\Phi}^{+}$, as recorded in $\S 3$ of [3], remain unchanged in the metaplectic setting. Indeed, they are formal consequences of the properties of induction and Jacquet functors which were proved in [2] in the setting of general $\ell$-groups. We also note that all six functors take genuine representations into genuine representations.

The modular characters which appear in the definitions of the $\Psi$ and $\Phi$ functors may all be expressed as powers of the character $|\operatorname{det}|$ restricted to various subgroups of $G(r)$. Since this character will occur frequently, we introduce the abbreviation $\mathrm{d}=|\operatorname{det}|$.

If $\tau \in \mathcal{R}_{\text {alg }}(\widetilde{P}(r))$, then, following [3], we define a sequence of representations $\tau^{(k)} \in$ $\mathcal{R}_{\text {alg }}(\widetilde{G}(r-k))$ by $\tau^{(k)}=\Psi^{-} \circ\left(\Phi^{-}\right)^{k-1}(\tau)$ for $k=1, \ldots, r$, and call $\tau^{(k)}$ the $k$ th derivative of $\tau$. If $\pi \in \mathcal{R}_{\text {alg }}(\widetilde{G}(r))$, then the $k$ th derivative of the representation $\left.\pi\right|_{\widetilde{P}(r)}$ is also referred to as the $k$ th derivative of $\pi$ and denoted by $\pi^{(k)}$. In this case the notation is naturally extended by setting $\pi^{(0)}=\pi$.

Generally, in later applications of the discussion in this section we shall be dealing with admissible representations of $\widetilde{G}(r)$ rather than with general algebraic representations. However, even if $\pi \in \mathcal{R}_{\text {alg }}(\widetilde{G}(r))$ is admissible, the representation $\left.\pi\right|_{\widetilde{P}(r)}$ will not generally be admissible and so we shall be forced to consider general algebraic representations of $\widetilde{P}(r)$. If $G$ is any $\ell$-group and $\tau_{1}, \tau_{2}, \tau_{3} \in \mathcal{R}_{\text {alg }}(G)$, then, for purely algebraic reasons, we have natural isomorphisms

$$
\operatorname{Hom}_{G}\left(\tau_{1} \otimes \tau_{2}, \widehat{\tau}_{3}\right) \cong \operatorname{Hom}_{G}\left(\tau_{1} \otimes \tau_{2} \otimes \tau_{3}, 1\right) \cong \operatorname{Hom}_{G}\left(\tau_{1} \otimes \tau_{3}, \widehat{\tau}_{2}\right) .
$$


The following propositions record the adjointness properties of the $\Psi$ and $\Phi$ functors and their behavior under the formation of contragredients.

Proposition 4.2. - Let $\rho \in \mathcal{R}_{\text {alg }}(\widetilde{G}(r-1)), \tau \in \mathcal{R}_{\text {alg }}(\widetilde{P}(r))$ and $\kappa \in \mathcal{R}_{\text {alg }}(\widetilde{P}(r-1))$. Then:

1. $\operatorname{Hom}_{\widetilde{P}(r)}\left(\tau, \Psi^{+}(\rho)\right) \cong \operatorname{Hom}_{\widetilde{G}(r-1)}\left(\Psi^{-}(\tau), \rho\right) ;$

2. $\operatorname{Hom}_{\widetilde{P}(r)}\left(\Psi^{+}(\rho), \tau\right) \cong \operatorname{Hom}_{\widetilde{G}(r-1)}\left(\rho, \widehat{\Psi}^{-}(\tau)\right)$;

3. $\operatorname{Hom}_{\widetilde{P}(r)}\left(\Phi^{+}(\kappa), \tau\right) \cong \operatorname{Hom}_{\widetilde{P}(r-1)}\left(\kappa, \Phi^{-}(\tau)\right)$;

4. $\operatorname{Hom}_{\widetilde{P}(r-1)}\left(\Phi^{-}(\tau), \kappa\right) \cong \operatorname{Hom}_{\widetilde{P}(r)}\left(\tau, \widehat{\Phi}^{+}(\kappa)\right)$.

All the implied maps underlie natural transformations.

Proof. - Statements 1, 3 and 4 are in [3], Proposition 3.2. Since $Y_{r}^{*}$ acts trivially on $\Psi^{+}(\rho)$, the image of any homomorphism from $\Psi^{+}(\rho)$ to $\tau$ is contained in the space of $\widehat{\Psi}^{-}(\tau)$. Thus the map from the right-hand side of statement 2 to the left given by composition with the inclusion $\widehat{\Psi}^{-}(\tau) \hookrightarrow \tau$ yields the required isomorphism. This makes the naturality claim clear.

Proposition 4.3. - Let $\rho \in \mathcal{R}_{\text {alg }}(\widetilde{G}(r-1)), \tau \in \mathcal{R}_{\text {alg }}(\widetilde{P}(r))$ and $\kappa \in \mathcal{R}_{\text {alg }}(\widetilde{P}(r-1))$. Then:

1. $\widehat{\Psi^{+}(\rho)} \cong \mathrm{d}^{-1} \otimes \Psi^{+}(\widehat{\rho})$

2. $\widehat{\Psi^{-}(\tau)} \cong \mathrm{d} \otimes \widehat{\Psi}^{-}(\widehat{\tau})$;

3. $\widehat{\Phi^{+}(\kappa)} \cong \mathrm{d}^{-1} \otimes \widehat{\Phi}^{+}(\mathrm{d} \otimes \widehat{\kappa})$;

4. $\widehat{\Phi^{-}(\tau)} \cong \Phi^{-}(\widehat{\tau})$.

The implied maps in statements 1 and 2 underlie natural transformations.

Proof. - Statements 1 and 3 are in [3], Proposition 3.4 except for the naturality claim for 1; however, this follows immediately from the proof. The proofs of 2 and 4 are similar.

\section{Exceptional representations}

In [12] the notion of an exceptional representation of $\widetilde{G}(r)$ is introduced and the fundamental properties of these representations are established. Our first task here is to extend this notion to the group $\widetilde{G}(\gamma)$ for any $\gamma \in \mathbb{P}$, as was suggested, but not systematically pursued, in [5]. Having the metaplectic tensor product available will prove particularly convenient in this connection. Secondly, we shall compute the derivatives of an exceptional representation of $\widetilde{G}(r)$. As far as the author is aware, the first derivative has not previously been considered. It is shown in [5] that the second derivative is an exceptional representation of $\widetilde{G}(r-2)$; we shall identify it precisely. Subject to a presumably unnecessary technical hypothesis, the remaining derivatives are shown to be zero.

We begin with two technical lemmas. If $\pi$ is a representation of any $\ell$-group and $\pi$ has a chain

$$
\pi=\pi_{k} \geqslant \pi_{k-1} \geqslant \cdots \geqslant \pi_{1} \geqslant \pi_{0}=0
$$

of subrepresentations such that $\pi_{i} / \pi_{i-1} \cong \tau_{i}$ for $i=1, \ldots, k$, then we shall follow [3] in saying that $\pi$ is glued from the representations $\tau_{1}, \ldots, \tau_{k}$.

LEMma 5.1. - Let $\gamma, \delta \in \mathbb{P}$ with $\gamma \leqslant \delta$ and $|\delta|=r$ and suppose that $\rho$ is an algebraic representation of $\widetilde{G}\left(\gamma_{0}\right)$. Then $\varphi_{\delta, \gamma}\left(i_{\gamma_{0}, \delta}(\rho)\right)$ is glued from the representations $i_{\gamma_{0}, \gamma}\left({ }^{w} \rho\right)$ as $w$ runs over $W(\gamma) \backslash W(\delta)$. 
Proof. - The usual Bruhat decomposition of $G(\delta)$ with respect to the parabolic subgroup $G(\gamma) \cdot N(\delta, \gamma)$ lifts to a decomposition of $\widetilde{G}(\delta)$. Given this observation, the lemma is simply a version of Bernstein's and Zelevinsky's geometric lemma and it follows from Theorem 5.2 of [3] just as in that paper.

LEMMA 5.2. - Let $\gamma, \delta \in \mathbb{P}$ with $\gamma \leqslant \delta$ and $|\delta|=r$ and suppose that $w \in W(\delta)$ is such that ${ }^{w} \mu_{\delta, \gamma_{0}}=\mu_{\delta, \gamma_{0}}$ on the group $Z_{2}(\gamma)$. Then $w \in W(\gamma)$.

Proof. - This follows from a routine combinatorial argument based upon the explicit expression for $\mu_{\delta, \gamma_{0}}$.

It follows from (2.3) that $\widetilde{G}(1) \cong F^{\times} \times \mu_{2}$ and hence every character $\chi$ of $F^{\times}$gives rise to a genuine character of $\widetilde{G}(1)$; we shall use the same symbol to denote both objects. If $\chi$ is such a character, then $\chi^{[r]}=\left(\left.\chi\right|_{\widetilde{G}_{2}(1)}\right) \otimes \cdots \otimes\left(\left.\chi\right|_{\widetilde{G}_{2}(1)}\right)(r$ factors $)$ is a genuine character of $\widetilde{G}_{2}\left(\gamma_{0}\right)$. We may choose a character $\omega$ of $Z(\widetilde{G}(r))$ which satisfies

$$
\left.\omega\right|_{\widetilde{Z}_{2}(r)}=\left.\chi^{[r]}\right|_{\widetilde{Z}_{2}(r)} .
$$

When $\omega$ and $\chi$ as above satisfy (5.1), then we shall call them compatible. For any compatible $\chi$ and $\omega$ we set $\chi_{r, \omega}=\chi \widetilde{\otimes}_{\omega} \chi \widetilde{\otimes} \cdots \widetilde{\otimes} \chi(r$ factors $)$. This is a genuine irreducible representation of $\widetilde{G}\left(\gamma_{0}\right)$. We remark that, in contrast to the usual tensor product, the metaplectic tensor product of one-dimensional representations is not usually one-dimensional.

We claim that ${ }^{w} \chi_{r, \omega} \cong \chi_{r, \omega}$ for all $w \in W$. It suffices to verify this when $w=s_{\alpha}$ for some simple root $\alpha$. In order to see this we first note that ${ }^{w} \omega=\omega$, so that $Z(\widetilde{G}(r))$ acts on ${ }^{w} \chi_{r, \omega}$ and $\chi_{r, \omega}$ via the same character. Then the associativity of the metaplectic tensor product (Proposition 3.5) reduces us to the case of two factors and, in this case, the claim is clear directly from the definition.

For any $\gamma \in \mathbb{P}$ with $|\gamma|=r$ and any compatible characters $\omega$ and $\chi$ we define

$$
\pi_{\gamma}(\chi, \omega)=i_{\gamma_{0}, \gamma}\left(\mu_{\gamma, \gamma_{0}}^{-1 / 4} \otimes \chi_{r, \omega}\right)
$$

Proposition 5.1. - Let $\gamma, \delta \in \mathbb{P}$ satisfy $\gamma \leqslant \delta$ and $|\delta|=r$. Then

$$
\varphi_{\delta, \gamma}\left(\pi_{\delta}(\chi, \omega)\right) \cong \bigoplus_{w \in W(\gamma) \backslash W(\delta)} i_{\gamma_{0}, \gamma}\left({ }^{w} \mu_{\delta, \gamma_{0}}^{-1 / 4} \otimes \chi_{r, \omega}\right) .
$$

Proof. - From Lemma 5.1 and the fact that ${ }^{w} \chi_{r, \omega} \cong \chi_{r, \omega}$ it follows that $\varphi_{\delta, \gamma}\left(\pi_{\delta}(\chi, \omega)\right)$ is glued from the indicated constituents. Lemma 5.2 implies that the $\widetilde{Z}_{2}(\gamma)$ characters of these constituents are all distinct and hence there are no non-trivial extensions between them.

Suppose that $\eta$ is a character of $\widetilde{G}_{2}\left(\gamma_{0}\right)$. Then, following [12], we let $\eta^{2}$ be the character of $G\left(\gamma_{0}\right)$ defined by $\eta^{2}(h)=\eta\left(\mathbf{s}\left(h^{2}\right)\right)$. From any character $\lambda$ of $G\left(\gamma_{0}\right)$ and $\operatorname{root} \alpha=(i, j)$ of $G(r)$ we obtain a character $\lambda_{\alpha}$ of $F^{\times}$by setting $\lambda_{\alpha}(x)=\lambda\left(h_{\alpha}(x)\right)$ where

$$
h_{\alpha}(x)=\operatorname{diag}\left(1, \ldots, 1, \underset{i}{x}, 1, \ldots, 1, \underset{j}{x}{ }^{-1}, 1, \ldots, 1\right) .
$$

A character $\eta$ of $\widetilde{G}_{2}\left(\gamma_{0}\right)$ (or of $Z(\widetilde{G}(r)) \cdot \widetilde{G}_{2}\left(\gamma_{0}\right)$ ) is called exceptional by Kazhdan and Patterson if it satisfies $\eta_{\alpha}^{2}=|\cdot|$ for all $\alpha \in \Delta$. 
If we denote by $w_{0}$ the longest element of $W$ with respect to our chosen positive system then it is routine to check that $w_{0}\left(\mu_{(r), \gamma_{0}}^{-1 / 4} \otimes \chi^{[r]}\right)$ is an exceptional character. Knowing this, Theorem I.2.9 of [12] implies that, for every pair of compatible characters $\chi$ and $\omega$, the representation $\pi_{(r)}(\chi, \omega)$ has a unique irreducible subrepresentation. This subrepresentation is isomorphic to the unique irreducible quotient of

$$
i_{\gamma_{0},(r)}\left(w_{0}\left(\mu_{(r), \gamma_{0}}^{-1 / 4} \otimes \chi_{r, \omega}\right)\right) \cong i_{\gamma_{0},(r)}\left(\mu_{(r), \gamma_{0}}^{1 / 4} \otimes \chi_{r, \omega}\right)
$$

and is called by Kazhdan and Patterson an exceptional representation. We shall denote the unique irreducible subrepresentation of $\pi_{(r)}(\chi, \omega)$ by $\vartheta_{(r)}(\chi, \omega)$. For any $\gamma \in \mathbb{P}$ satisfying $|\gamma|=r$ we define

$$
\vartheta_{\gamma}(\chi, \omega)=\mu_{(r), \gamma}^{1 / 4} \otimes \varphi_{(r), \gamma}\left(\vartheta_{(r)}(\chi, \omega)\right)
$$

and extend the scope of the term "exceptional representation" to include all the $\vartheta_{\gamma}(\chi, \omega)$.

Although it has been convenient to allow arbitrary characters $\chi$ up to this point, changing $\chi$ is equivalent to twisting the associated representation by a character and so no information is lost if we restrict $\chi$ to be the trivial character. If $\omega$ is a character of $Z(\widetilde{G}(r))$ which is compatible with the trivial character then we shall call $\omega$ suitable. If $\omega_{1}$ and $\omega_{2}$ are suitable characters, then $\omega_{1} \cdot \omega_{2}^{-1}$ is trivial on $\widetilde{Z}_{2}(r)$ and it easily follows that there is a unique suitable character if $r$ is even and that there are $\left[F^{\times}:\left(F^{\times}\right)^{2}\right]$ suitable characters if $r$ is odd. If $\gamma \in \mathbb{P}$ and $\omega$ is a suitable character then we shall write $\vartheta_{\gamma, \omega}$ in place of $\vartheta_{\gamma}\left(\chi_{0}, \omega\right)$.

We remark that when $r$ is odd the various exceptional representations $\vartheta_{\gamma, \omega}$ for suitable characters $\omega$ are all twists of one another. However, there doesn't seem to be a canonical way of choosing one of the twists.

THEOREM 5.1. - Let $\omega$ be a suitable character and $\gamma, \delta \in \mathbb{P}$ be such that $\gamma \leqslant \delta$ and $|\delta|=r$.

1. We have $\varphi_{\delta, \gamma}\left(\vartheta_{\delta, \omega}\right) \cong \mu_{\delta, \gamma}^{-1 / 4} \otimes \vartheta_{\gamma, \omega}$.

2. There is an isomorphism $\vartheta_{\gamma_{0}, \omega} \cong \chi_{r, \omega}$.

3. The representation $\vartheta_{\gamma, \omega}$ is isomorphic to the unique irreducible subrepresentation of $\pi_{\gamma}\left(\chi_{0}, \omega\right)$.

4. If $\gamma=\left(\gamma_{1}, \gamma_{2}\right)$, then $\vartheta_{\gamma, \omega} \cong \vartheta_{\gamma_{1}, \omega_{1}} \widetilde{\otimes}_{\omega} \vartheta_{\gamma_{2}, \omega_{2}}$, where $\omega_{j}$ is a suitable character of $Z\left(\widetilde{G}\left(\left|\gamma_{j}\right|\right)\right)$ for $j=1,2$.

5. The contragredient of $\vartheta_{\gamma, \omega}$ is isomorphic to $\vartheta_{\gamma, \omega^{-1}}$.

6. The representation $\vartheta_{\delta, \omega}$ is isomorphic to the unique irreducible subrepresentation of $i_{\gamma, \delta}\left(\mu_{\delta, \gamma}^{-1 / 4} \otimes \vartheta_{\gamma, \omega}\right)$.

Proof. -1 . This is an easy consequence of the definition of $\vartheta_{\delta, \omega}$.

2. When rewritten in our terminology, the so-called Periodicity Theorem (Theorem I.2.9(e) of [12]) asserts that $\varphi_{(r), \gamma_{0}}\left(\vartheta_{(r), \omega}\right) \cong \mu_{(r), \gamma_{0}}^{-1 / 4} \otimes\left(\chi_{0}\right)_{r, \omega}$. Combining this isomorphism with the definition gives part 2 .

3. Using parts 1 and 2 and the Frobenius reciprocity law we obtain

$$
\operatorname{Hom}_{\widetilde{G}(\gamma)}\left(\vartheta_{\gamma, \omega}, \pi_{\gamma}\left(\chi_{0}, \omega\right)\right) \cong \operatorname{Hom}_{\widetilde{G}\left(\gamma_{0}\right)}\left(\mu_{\gamma, \gamma_{0}}^{-1 / 4} \otimes\left(\chi_{0}\right)_{r, \omega}, \mu_{\gamma, \gamma_{0}}^{-1 / 4} \otimes\left(\chi_{0}\right)_{r, \omega}\right) .
$$

The identity map from $\mu_{\gamma, \gamma_{0}}^{-1 / 4} \otimes\left(\chi_{0}\right)_{r, \omega}$ to itself corresponds under this isomorphism to an embedding of $\vartheta_{\gamma, \omega}$ into $\pi_{\gamma}\left(\chi_{0}, \omega\right)$. Thus $\vartheta_{\gamma, \omega}$ may be regarded as a subrepresentation of $\pi_{\gamma}\left(\chi_{0}, \omega\right)$. Now, $\pi_{\gamma}\left(\chi_{0}, \omega\right)$ is an induced representation and it follows from the metaplectic analogue of [3], Theorem 2.4 (a) and (d) that $\pi_{\gamma}\left(\chi_{0}, \omega\right)$ has no cuspidal constituents. In 
particular, $\vartheta_{\gamma, \omega}$ has no cuspidal constituents and, since $\varphi_{\gamma, \gamma_{0}}\left(\vartheta_{\gamma, \omega}\right) \cong \mu_{\gamma, \gamma_{0}}^{-1 / 4} \otimes\left(\chi_{0}\right)_{r, \omega}$ is irreducible, it follows that $\vartheta_{\gamma, \omega}$ is irreducible. Finally, we must show that $\pi_{\gamma}\left(\chi_{0}, \omega\right)$ has no other irreducible subrepresentations. So let $\rho \leqslant \pi_{\gamma}\left(\chi_{0}, \omega\right)$ be an irreducible representation other than $\vartheta_{\gamma, \omega}$. Using Proposition 5.1, the fact that Jacquet functors are exact and the observations just made, we see that $\varphi_{\gamma, \gamma_{0}}(\rho) \cong{ }^{w} \mu_{\gamma, \gamma_{0}}^{-1 / 4} \otimes\left(\chi_{0}\right)_{r, \omega}$ for some $w \in W(\gamma) \backslash\{1\}$. The transitivity of induction implies that $\pi_{(r)}\left(\chi_{0}, \omega\right) \cong i_{\gamma,(r)}\left(\mu_{(r), \gamma}^{-1 / 4} \otimes \pi_{\gamma}\left(\chi_{0}, \omega\right)\right)$ and so $i_{\gamma,(r)}\left(\mu_{(r), \gamma}^{-1 / 4} \otimes \rho\right)$ is a subrepresentation of $\pi_{(r)}\left(\chi_{0}, \omega\right)$. Since $\vartheta_{(r), \omega}$ is the unique irreducible subrepresentation of $\pi_{(r)}\left(\chi_{0}, \omega\right)$, it follows that $\operatorname{Hom}_{\widetilde{G}(r)}\left(\vartheta_{(r), \omega}, i_{\gamma,(r)}\left(\mu_{\gamma,(r)}^{-1 / 4} \otimes \rho\right)\right) \neq\{0\}$. However, this space is isomorphic to

$$
\operatorname{Hom}_{\widetilde{G}(\gamma)}\left(\mu_{(r), \gamma}^{-1 / 4} \otimes \vartheta_{\gamma, \omega}, \mu_{(r), \gamma}^{-1 / 4} \otimes \rho\right) \cong \operatorname{Hom}_{\widetilde{G}(\gamma)}\left(\vartheta_{\gamma, \omega}, \rho\right)
$$

and consequently $\vartheta_{\gamma, \omega} \cong \rho$. This isomorphism gives rise to a contradiction, since Lemma 5.2 shows that $\varphi_{\gamma, \gamma_{0}}\left(\vartheta_{\gamma, \omega}\right)$ is not isomorphic to $\varphi_{\gamma, \gamma_{0}}(\rho)$.

4. Using Propositions 4.1 and 3.5 we obtain $\varphi_{\gamma, \gamma_{0}}\left(\vartheta_{\gamma_{1}, \omega_{1}} \widetilde{\otimes}_{\omega} \vartheta_{\gamma_{2}, \omega_{2}}\right) \cong \mu_{\gamma, \gamma_{0}}^{-1 / 4} \otimes\left(\chi_{0}\right)_{r, \omega}$ and consequently the representation $\vartheta_{\gamma_{1}, \omega_{1}} \widetilde{\otimes}_{\omega} \vartheta_{\gamma_{2}, \omega_{2}}$ may be regarded as a subrepresentation of $\pi_{\gamma}\left(\chi_{0}, \omega\right)$. From Proposition 3.3 this representation is also irreducible and hence it is isomorphic to $\vartheta_{\gamma, \omega}$ by part 3 .

5. From Proposition 1.9 (d) of [3] and Proposition 3.4 we obtain the isomorphism $\pi_{(r)}\left(\chi_{0}, \omega\right)^{\wedge} \cong i_{\gamma_{0},(r)}\left(\mu_{(r), \gamma_{0}}^{1 / 4} \otimes\left(\chi_{0}\right)_{r, \omega}-1\right)$. We know that $\vartheta_{(r), \omega^{-1}}$ is the unique irreducible quotient of the right-hand representation in this isomorphism and that, under the pairing between $\pi_{(r)}\left(\chi_{0}, \omega\right)$ and its contragredient, the irreducible subrepresentation $\vartheta_{(r), \omega}$ must be paired with an irreducible quotient. Therefore $\widehat{\vartheta}_{(r), \omega} \cong \vartheta_{(r), \omega^{-1}}$. The general case follows on using part 4 and Proposition 3.4 .

6. From part 3 and the exactness of induction we know that the representation $i_{\gamma, \delta}\left(\mu_{\delta, \gamma}^{-1 / 4} \otimes\right.$ $\left.\vartheta_{\gamma, \omega}\right)$ may be regarded as a subrepresentation of $i_{\gamma, \delta}\left(\mu_{\delta, \gamma}^{-1 / 4} \otimes \pi_{\gamma}\left(\chi_{0}, \omega\right)\right) \cong \pi_{\delta}\left(\chi_{0}, \omega\right)$. The claim now follows from an application of part 3.

THEOREM 5.2. - Let $\omega_{r}$ be a suitable character of $Z(\widetilde{G}(r))$. If $r$ is odd, then

$$
\vartheta_{(r), \omega_{r}}^{(1)} \cong \mathrm{d}^{-1 / 4} \otimes \vartheta_{(r-1), \omega_{r-1}}
$$

where $\omega_{r-1}$ is the unique suitable character of $Z(\widetilde{G}(r-1))$. If $r$ is even, then

$$
\vartheta_{(r), \omega_{r}}^{(1)} \cong \bigoplus_{\omega_{r-1}} \mathrm{~d}^{-1 / 4} \otimes \vartheta_{(r-1), \omega_{r-1}},
$$

where the sum is over all suitable characters of $Z(\widetilde{G}(r-1))$.

Proof. - By definition, $\vartheta_{(r), \omega_{r}}^{(1)}=\Psi^{-}\left(\left.\vartheta_{(r), \omega_{r}}\right|_{\widetilde{P}(r)}\right)$ and, since Jacquet functors and restriction functors commute, this may be expressed alternatively as $\vartheta_{(r), \omega_{r}}^{(1)}=\left.\varphi_{(r),(r-1,1)}\left(\vartheta_{(r), \omega_{r}}\right)\right|_{\widetilde{G}(r-1)}$. By part 1 of Theorem 5.1 we have $\varphi_{(r),(r-1,1)}\left(\vartheta_{(r), \omega_{r}}\right) \cong \mu_{(r),(r-1,1)}^{-1 / 4} \otimes \vartheta_{(r-1,1), \omega_{r}}$ and using part 4 of the same result gives $\vartheta_{(r-1,1), \omega_{r}} \cong \vartheta_{(r-1), \omega_{r-1}} \widetilde{\otimes}_{\omega_{r}} \vartheta_{(1), \omega_{1}} \cong \vartheta_{(r-1), \omega_{r-1}} \widetilde{\otimes}_{\omega_{r}} \omega_{1}$, where $\omega_{1}$ is a suitable character of $Z(\widetilde{G}(1))=\widetilde{G}(1)$. We have $\mu_{(r),(r-1,1)}(g, 1)=\mathrm{d}(g)$ for $g \in G(r-1)$ and so it remains to evaluate $\left.\left(\vartheta_{(r-1), \omega_{r-1}} \widetilde{\otimes}_{\omega_{r}} \omega_{1}\right)\right|_{\widetilde{G}(r-1)}$. 
Suppose that $r$ is odd, so that $r-1$ is even. Let $\sigma$ be an irreducible summand of $\vartheta_{(r-1), \omega_{r-1}}^{2}$. Then, according to Corollary 3.1,

$$
\vartheta_{(r-1), \omega_{r-1}} \widetilde{\otimes}_{\omega_{r}} \omega_{1} \cong \operatorname{ind} \underset{\widetilde{G}^{2}(r-1)}{\widetilde{G}(r-1) \widetilde{\times} \widetilde{G}(1)}\left(\sigma \otimes \omega_{1}\right)
$$

and using the Mackey subgroup theorem to compute the restriction of this representation to $\widetilde{G}(r-1)$ we find that it is

$$
\left.\left(\vartheta_{(r-1), \omega_{r-1}} \widetilde{\otimes}_{\omega_{r}} \omega_{1}\right)\right|_{\widetilde{G}(r-1)} \cong \operatorname{ind}_{\widetilde{G}^{2}(r-1)} \widetilde{\widetilde{G}}(\sigma) \cong \vartheta_{(r-1), \omega_{r-1}},
$$

by Proposition 3.2. This proves the first isomorphism.

If $r$ is even then $r-1$ is odd and so Corollary 3.1 shows that

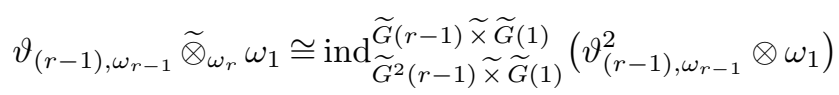

and proceeding as in the previous paragraph we find that the restriction of this to $\widetilde{G}(r-1)$ is isomorphic to $\bigoplus_{\chi \in\left(\widetilde{G}(r-1) / \widetilde{G}^{2}(r-1)\right)} \chi \chi \otimes \vartheta_{(r-1), \omega_{r-1}}$. This sum has the same terms as the one given in the statement.

In order to identify the second derivative of $\vartheta_{(r), \omega}$ precisely we require some preliminary observations. It follows from (2.6) that $\widetilde{Z}(r)$ centralizes every subgroup of $N^{*}(r)$. Consequently, if we apply a Jacquet functor with respect to any subgroup of $N^{*}(r)$ to a representation of $\widetilde{G}(r)$ then the result may be regarded as a representation of $\widetilde{Z}(r)$. This remark applies, in particular, to the derivatives. In order to make use of it, we next describe all genuine characters of $\widetilde{Z}(r)$.

If $t_{1}, t_{2} \in F^{\times}$then (2.3) shows that $\sigma_{r}\left(t_{1} I_{r}, t_{2} I_{r}\right)=\left(t_{1}, t_{2}\right)^{r(r-1) / 2}$ and it follows that $\widetilde{Z}(r) \cong \widetilde{G}(1) \cong F^{\times} \times \mu_{2}$ when $r \equiv 0,1(4)$. When $r \equiv 2,3(4), \widetilde{Z}(r)$ may be identified with the group $H$ with underlying set $F^{\times} \times \mu_{2}$ and multiplication law $\left[t_{1}, \varepsilon_{1}\right]\left[t_{2}, \varepsilon_{2}\right]=\left[t_{1} t_{2},\left(t_{1}, t_{2}\right) \varepsilon_{1} \varepsilon_{2}\right]$. We have already observed that, in the first case, the set of genuine characters of $\widetilde{Z}(r)$ is in oneto-one correspondence with the set of characters of $F^{\times}$. It is a remarkable fact that, after fixing an additive character $\psi$ of $F$, the same is true in the second case also. Recall that in [16], Weil associates to each additive character $\psi$ of $F$ a complex number $\gamma(\psi)$. If $\psi$ is such a character and $a \in F^{\times}$, then we put $\psi_{a}(x)=\psi(a x)$ for all $x \in F$. The essential property of $\gamma$ for our purposes is expressed by the equation $(a, b)=\gamma\left(\psi_{a b}\right) \gamma(\psi) \gamma\left(\psi_{a}\right)^{-1} \gamma\left(\psi_{b}\right)^{-1}$ for all $a, b \in F^{\times}$. Following [8] we introduce a function on $F^{\times}$by $\mu_{\psi}(a)=\gamma(\psi) / \gamma\left(\psi_{a}\right)$. With this notation, the above relation may be written as $(a, b)=\mu_{\psi}(a) \mu_{\psi}(b) \mu_{\psi}(a b)^{-1}$. If $\chi$ is a character of $F^{\times}$, then we define $\chi_{\psi}$ by $\chi_{\psi}([t, \varepsilon])=\chi(t) \mu_{\psi}(t) \varepsilon$ for $[t, \varepsilon] \in H$. One sees that $\chi_{\psi}$ is a character of $H$ and that all genuine characters of $H$ arise in this way.

What we actually require is the character via which $\widetilde{Z}(2)$ acts on $\vartheta_{(2), \omega_{2}}^{(2)}$, where $\omega_{2}$ is the unique suitable character of $Z(\widetilde{G}(2))$. Fortunately, this has already been computed by Gelbart and Piatetski-Shapiro.

LEMMA 5.3. - Let $\omega_{2}$ be the unique suitable character of $Z(\widetilde{G}(2))$. Then $\vartheta_{(2), \omega_{2}}^{(2)}$ is onedimensional and $\widetilde{Z}(2)$ acts on it via the character $\left(\chi_{0}\right)_{\psi}$, where $\psi$ is the additive character of $F$ with respect to which the derivative is formed.

Proof. - This is Theorem 2.2 of [8]. Note that it follows from the definition of $\vartheta_{(2), \omega_{2}}$ together with Proposition 2.3.3 of [8] that $\vartheta_{(2), \omega_{2}}$ is the representation which Gelbart and PiatetskiShapiro denote by $r_{\chi_{0}}$. 
THEOREM 5.3. - Let $\omega_{r}$ be a suitable character of $Z(\widetilde{G}(r))$. Then

$$
\vartheta_{(r), \omega_{r}}^{(2)} \cong \mathrm{d}^{-1 / 2} \otimes \vartheta_{(r-2), \omega_{r-2}}
$$

where $\omega_{r-2}$ is related to $\omega_{r}$ by the equation

$$
\omega_{r}=\left.\left(\omega_{r-2} \otimes\left(\chi_{0}\right)_{\psi}\right)\right|_{Z(\widetilde{G}(r))},
$$

where $\psi$ is the additive character of $F$ with respect to which the derivative is formed.

Proof. - The second derivative functor factors through $\varphi_{(r),(r-2,2)}$ and we begin with the isomorphisms $\varphi_{(r),(r-2,2)}\left(\vartheta_{(r), \omega_{r}}\right) \cong \mu_{(r),(r-2,2)}^{-1 / 4} \otimes \vartheta_{(r-2,2), \omega_{r}}$ and

$$
\vartheta_{(r-2,2), \omega_{r}} \cong \vartheta_{(r-2), \nu} \widetilde{\otimes}_{\omega_{r}} \vartheta_{(2), \omega_{2}}
$$

which are furnished by Theorem 5.1. Here $\nu$ is any suitable character of $Z(\widetilde{G}(r-2))$ and $\omega_{2}$ is as in Lemma 5.3. For $g \in \widetilde{G}(r-2)$ we have $\mu_{(r),(r-2,2)}(g)=|\operatorname{det}(g)|^{2}$ and so it remains to apply the Jacquet functor $\mathcal{R}_{\text {alg }}(\widetilde{G}(r-2) \widetilde{\times} \widetilde{G}(2)) \rightarrow \mathcal{R}_{\text {alg }}(\widetilde{G}(r-2))$ which corresponds to the character $\psi$ of $N^{*}(2)$ to the right-hand side of (5.4). This may be done in stages by first restricting the representation to $\widetilde{G}(r-2) \widetilde{\times} \widetilde{G}^{2}(2)$ and then applying the Jacquet functor with respect to $\psi$ in the second factor.

By Corollary 3.1 we may choose an irreducible subrepresentation $\sigma$ of $\vartheta_{(2), \omega_{2}}^{2}$ so that

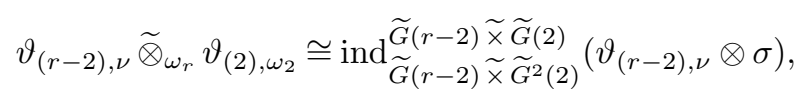

and hence

$$
\begin{aligned}
\left.\left(\vartheta_{(r-2), \nu} \widetilde{\otimes}_{\omega_{r}} \vartheta_{(2), \omega_{2}}\right)\right|_{\widetilde{G}(r-2)} \widetilde{\times} \widetilde{G}^{2}(2) & \cong \bigoplus_{g \in \widetilde{G}(2) / \widetilde{G}^{2}(2)}{ }^{g}\left(\vartheta_{(r-2), \nu} \otimes \sigma\right) \\
& \cong \bigoplus_{g \in \widetilde{G}(2) / \widetilde{G}^{2}(2)}\left(\chi_{g} \otimes \vartheta_{(r-2), \nu}\right) \otimes{ }^{g} \sigma,
\end{aligned}
$$

where, as before, $\chi_{g}(h)=(\operatorname{det}(g)$, $\operatorname{det}(h))$. Since $r$ and $r-2$ have the same parity, $Z(\widetilde{G}(r)) \subseteq$ $Z(\widetilde{G}(r-2)) \widetilde{\times} \widetilde{Z}(2)$ and (5.5) implies that, for all $g \in \widetilde{G}(2) / \widetilde{G}^{2}(2)$, we have

$$
\omega_{r}=\left.\left(\left(\chi_{g} \otimes \nu\right) \otimes \omega_{\left(g_{\sigma}\right)}\right)\right|_{Z(\widetilde{G}(r))} .
$$

From Lemma 5.3 we know that $\vartheta_{(2), \omega_{2}}^{(2)}$ is one-dimensional and that $\widetilde{Z}(2)$ acts on it via $\left(\chi_{0}\right)_{\psi}$. Thus, after applying the $\left(N^{*}(2), \psi\right)$ Jacquet functor, all the summands in (5.5) give zero except for the one which satisfies $\omega_{\left(g_{\sigma}\right)}=\left(\chi_{0}\right)_{\psi}$. This remaining summand yields the representation $\chi_{g} \otimes \vartheta_{(r-2), \nu}$. This, in turn, is isomorphic to $\vartheta_{(r-2), \omega_{r-2}}$ where, according to (5.6), $\omega_{r-2}$ satisfies (5.3).

In the following theorem the assumption that $F$ is not dyadic is expected to be unnecessary. That it is so would follow from a fully elaborated theory of the trace formula for the group $\widetilde{G}(r)$ ( $c f$. the remarks in Section 4 of [7]). At present it seems that the necessary facts are known only when $r \leqslant 3$ but, when they are established in general, the hypothesis may be removed. The 
restriction to non-dyadic $F$ in the next section enters only through this theorem and so it could be removed at the same time.

THEOREM 5.4. - Let $\omega$ be a suitable character of $Z(\widetilde{G}(r))$. Then $\vartheta_{(r), \omega}^{(3)}=0$. If F is not dyadic, then $\vartheta_{(r), \omega}^{(k)}=0$ for $3 \leqslant k \leqslant r$.

Proof. - Since the $k$ th derivative functor factors through $\varphi_{(r),(r-k, k)}$ and $\varphi_{(r),(r-k, k)}\left(\vartheta_{(r), \omega}\right)$ is a twist of $\vartheta_{(r-k), \omega_{r-k}} \widetilde{\otimes}_{\omega} \vartheta_{(k), \omega_{k}}$ by Theorem 5.1, we are reduced to showing that $\vartheta_{(k), \omega_{k}}^{(k)}=0$. For $k=3$ and any $F$ this is Lemma 6 in Section 4 of [7]. Assuming $F$ is non-dyadic, we instead use Theorem I.3.5 of [12]. To see how it applies, observe that $\vartheta_{(k), \omega_{k}}^{(k)}$ is the space of Whittaker functionals on $\vartheta_{(k), \omega_{k}}$. According to [12], it remains to show that the symmetric group on $k$ letters has no free orbits on $(\mathbb{Z} / 2 \mathbb{Z})^{k}$ when $k \geqslant 3$. This follows at once from the pidgeonhole principle.

\section{The main results}

Throughout this section, $\omega$ and $\nu$ will be suitable characters of $Z(\widetilde{G}(r))$. The burden of notation will be quite heavy and, in order to lighten it as far as possible, we shall denote the exceptional representation $\vartheta_{(r), \omega}$ by $\vartheta_{r, \omega}$ if $\omega$ must be specified and by $\vartheta_{r}$ if not. Since all exceptional representations are genuine, $\mu_{2}$ acts trivially on the inner tensor product $\vartheta_{r, \omega} \otimes \vartheta_{r, \nu}$ which may therefore be viewed as a representation of $G(r)$. As explained in the introduction, we shall study the space of $G(r)$-invariant linear functionals on $\vartheta_{r, \omega} \otimes \vartheta_{r, \nu} \otimes \pi$ for $\pi$ a representation of $G(r)$. This space will be denoted by $\mathcal{L}(\omega, \nu ; \pi)$, abbreviated to $\mathcal{L}(\omega ; \pi)$ if $\omega=\nu$.

For the reasons discussed at the end of the previous section we must presently assume that $F$ is not a dyadic field or that $r=2$ or 3 . For the rest of this section we assume, without further notice, that one of these two conditions holds true.

If $\pi \in \mathcal{R}_{\text {alg }}(G(r))$ then we shall refer to $\pi^{(1)}, \ldots, \pi^{(r-1)}$ as the intermediate derivatives of $\pi$ (that is, intermediate between $\pi^{(0)}=\pi$ and the last derivative $\pi^{(r)}$ ).

Definition 6.1. - Let $\pi \in \mathcal{R}_{\text {alg }}(G(r))$ and $s \in \mathbb{C}$. We say that $\pi$ is general with respect to $s$ if no non-zero subquotient of any intermediate derivative of $\pi$ has central character, $\alpha$, satisfying $\alpha^{2}=\mathrm{d}^{-2 s-1 / 2}$.

It will be convenient to make a couple of simple observations which will be used repeatedly in what follows. First, the non-genuine character $\omega \cdot \nu$ is trivial on $Z_{2}(r)$ and hence we always have $(\omega \cdot \nu)^{-1}=\omega \cdot \nu$ regardless of the parity of $r$. Note that when $r$ is odd it isn't necessarily true that $\omega^{-1}=\omega$. Secondly, the restriction of the modular character $\mu_{(r),(r-1,1)}$ to $P(r)$ is equal to $\mathrm{d}$. Finally, we remark that if $\gamma \in \mathbb{P}$ then $\mu_{(r), \gamma}$, which we have previously defined to be a character of $\widetilde{G}(\gamma)$, extends to a character of $\widetilde{Q}(\gamma)$ by declaring it to be trivial on $N^{*}(\gamma)$. The extension is equal to the modular character of the group $\widetilde{Q}(\gamma)$. We shall use the same symbol for the extended character.

THEOREM 6.1. - Suppose that $s \in \mathbb{C}$ and $\pi$ is a homogeneous admissible representation of $G(r)$ of finite length which is general with respect to $s$. Then the dimension of the space of $G(r)$-invariant functionals on the representation

$$
\vartheta_{r, \omega} \otimes i_{(r-1,1),(r)}\left(\mu_{(r),(r-1,1)}^{s} \otimes \vartheta_{(r-1,1), \nu}\right) \otimes \pi
$$

is at most the dimension of the space of Whittaker functionals on $\pi$.

$4^{\text {e }}$ SÉRIE - TOME $34-2001-\mathrm{N}^{\circ} 5$ 
Proof. - The proof will be a variation on the proof of Theorem 5.1 in [5], keeping track of the exceptional parameter values. If $(\omega \cdot \nu) \otimes \omega_{\pi}$ is not trivial on $p_{r}(Z(\widetilde{G}(r)))$, then the space in question is zero and the claim is clear. We assume from now on that $(\omega \cdot \nu) \otimes \omega_{\pi}$ is trivial on $p_{r}(Z(\widetilde{G}(r)))$.

Suppose that $r$ is odd. Then the space of $G(r)$-invariant functionals is

$$
\begin{aligned}
& \operatorname{Hom}_{\widetilde{G}(r)}\left(\vartheta_{r, \omega} \otimes i_{(r-1,1),(r)}\left(\mu_{(r),(r-1,1)}^{s} \otimes \vartheta_{(r-1,1), \nu}\right) \otimes \pi, 1\right) \\
& \quad \cong \operatorname{Hom}_{\widetilde{G}(r)}\left(\vartheta_{r, \omega} \otimes \pi, i_{(r-1,1),(r)}\left(\mu_{(r),(r-1,1)}^{-s} \otimes \vartheta_{(r-1,1), \nu^{-1}}\right)\right) \\
& \cong \operatorname{Hom}_{\widetilde{Q}(r-1,1)}\left(\vartheta_{r, \omega} \otimes \pi, \mu_{(r),(r-1,1)}^{-s} \otimes \Psi^{+}\left(\vartheta_{(r-1,1), \nu^{-1}}\right)\right) .
\end{aligned}
$$

Since $r$ is odd, the representations in the first and second places in (6.1) have the same character under $\widetilde{Z}(r)$ and, because $\widetilde{Q}(r-1,1)=\widetilde{Z}(r) \cdot \widetilde{P}(r)$, the space in (6.1) is isomorphic to

$$
\operatorname{Hom}_{\widetilde{P}(r)}\left(\vartheta_{r, \omega} \otimes \pi, \mathrm{d}^{-s} \otimes \Psi^{+}\left(\left.\vartheta_{(r-1,1), \nu^{-1}}\right|_{\widetilde{G}(r-1)}\right)\right) .
$$

But $(r-1)$ is even and so $\vartheta_{(r-1,1), \nu^{-1}} \cong \vartheta_{r-1, \nu_{r-1}}$, where $\nu_{r-1}$ is the unique suitable character of $Z(\widetilde{G}(r-1))$. Thus $(6.2)$ is isomorphic to

$$
\operatorname{Hom}_{\widetilde{P}(r)}\left(\vartheta_{r, \omega} \otimes \pi, \mathrm{d}^{-s} \otimes \Psi^{+}\left(\vartheta_{r-1, \nu_{r-1}}\right)\right)
$$

If $r$ is even then we must reach (6.3) by a slightly different route. In this case $(r-1)$ is odd and so

$$
\vartheta_{(r-1,1), \nu^{-1}} \cong \operatorname{ind} \underset{\widetilde{G}(r-1)}{\widetilde{\times}(r-1) \widetilde{\times} \widetilde{G}(1)} \widetilde{G^{2}(1)}\left(\vartheta_{r-1, \nu_{r-1}} \otimes \chi_{0}\right),
$$

where $\nu_{r-1}$ is any suitable character of $Z(\widetilde{G}(r-1))$ and $\chi_{0}$ denotes the genuine character of $\widetilde{G}^{2}(1)$ which corresponds to the trivial character of $F^{\times}$. Thus, if we set $\widetilde{Q}_{2}(r-1,1)=$ $\widetilde{Z}_{2}(r) \cdot \widetilde{P}(r)$, then by the transitivity of induction we have

$$
i_{(r-1,1),(r)}\left(\mu_{(r),(r-1,1)}^{s} \otimes \vartheta_{(r-1,1), \nu^{-1}}\right) \cong \operatorname{ind}_{\widetilde{Q}_{2}(r-1,1)} \widetilde{G}_{(r)}\left(\mu_{(r),(r-1,1)}^{s} \otimes \vartheta_{r-1, \nu_{r-1}} \otimes \chi_{0}\right)
$$

and repeating the steps which led to (6.1) starting from this representation we find that the space of $G(r)$-invariant functionals is isomorphic to

$$
\operatorname{Hom}_{\widetilde{Q}_{2}(r-1,1)}\left(\vartheta_{r, \omega} \otimes \pi, \mu_{(r),(r-1,1)}^{-s} \otimes \Psi^{+}\left(\vartheta_{r-1, \nu_{r-1}} \otimes \chi_{0}\right)\right) .
$$

Since the $\widetilde{Z}_{2}(r)$-characters of the representations in the first and second places in this Hom-space agree, we find that it is again isomorphic to (6.3).

This completes the first part of the proof. From this point onwards the suitable characters with respect to which the exceptional representations are formed will not play a significant role and we shall allow ourselves to omit them from the notation.

For $0 \leqslant k \leqslant r-1$ and $z \in \mathbb{C}$ we shall consider the space

$$
\mathcal{H}_{k}(\pi, z)=\operatorname{Hom}_{\widetilde{P}(r-k)}\left(\Psi^{+}\left(\vartheta_{r-k-1}\right) \otimes\left(\Phi^{-}\right)^{k}(\pi) \otimes \vartheta_{r-k}, \mathrm{~d}^{z}\right)
$$

The space (6.3), whose dimension we wish to estimate, is isomorphic to $\mathcal{H}_{0}(\pi, 1-s)$. This may be seen by using part 5 of Theorem 5.1, (4.7) and part 1 of Proposition 4.3. 
As recorded in Section 3 of [3], we have a short exact sequence

$$
0 \longrightarrow \Phi^{+} \Phi^{-}\left(\vartheta_{r-k}\right) \longrightarrow \vartheta_{r-k} \longrightarrow \Psi^{+} \Psi^{-}\left(\vartheta_{r-k}\right) \longrightarrow 0
$$

and, since the tensor product yields an exact functor on the category of vector spaces, we obtain from this a short exact sequence

$$
\begin{aligned}
0 \longrightarrow \Psi^{+}\left(\vartheta_{r-k-1}\right) \otimes\left(\Phi^{-}\right)^{k}(\pi) \otimes \Phi^{+} \Phi^{-}\left(\vartheta_{r-k}\right) \\
\longrightarrow \Psi^{+}\left(\vartheta_{r-k-1}\right) \otimes\left(\Phi^{-}\right)^{k}(\pi) \otimes \vartheta_{r-k} \\
\longrightarrow \Psi^{+}\left(\vartheta_{r-k-1}\right) \otimes\left(\Phi^{-}\right)^{k}(\pi) \otimes \Psi^{+} \Psi^{-}\left(\vartheta_{r-k}\right) \longrightarrow 0 .
\end{aligned}
$$

Using this sequence and the definition of $\mathcal{H}_{k}(\pi, z)$ we obtain an exact sequence

$$
\begin{aligned}
0 \longrightarrow \operatorname{Hom}_{\widetilde{P}(r-k)}\left(\Psi^{+}\left(\vartheta_{r-k-1}\right) \otimes\left(\Phi^{-}\right)^{k}(\pi) \otimes \Psi^{+} \Psi^{-}\left(\vartheta_{r-k}\right), \mathrm{d}^{z}\right) \\
\longrightarrow \mathcal{H}_{k}(\pi, z) \\
\longrightarrow \operatorname{Hom}_{\widetilde{P}(r-k)}\left(\Psi^{+}\left(\vartheta_{r-k-1}\right) \otimes\left(\Phi^{-}\right)^{k}(\pi) \otimes \Phi^{+} \Phi^{-}\left(\vartheta_{r-k}\right), \mathrm{d}^{z}\right) .
\end{aligned}
$$

Now

$$
\begin{aligned}
& \operatorname{Hom}_{\widetilde{P}(r-k)}\left(\Psi^{+}\left(\vartheta_{r-k-1}\right) \otimes\left(\Phi^{-}\right)^{k}(\pi) \otimes \Psi^{+} \Psi^{-}\left(\vartheta_{r-k}\right), \mathrm{d}^{z}\right) \\
& \cong \operatorname{Hom}_{\widetilde{P}(r-k)}\left(\Psi^{+}\left(\vartheta_{r-k-1}\right) \otimes\left(\Phi^{-}\right)^{k}(\pi), \mathrm{d}^{z-1} \otimes \Psi^{+}\left(\widetilde{\left.\Psi^{-\left(\vartheta_{r-k}\right.}\right)}\right)\right) \\
& \cong \operatorname{Hom}_{\widetilde{G}(r-k-1)}\left(\Psi^{-}\left(\Psi^{+}\left(\vartheta_{r-k-1}\right) \otimes\left(\Phi^{-}\right)^{k}(\pi)\right), \mathrm{d}^{z-3 / 4} \otimes \oplus \vartheta_{r-k-1}\right) \\
& \cong \operatorname{Hom}_{\widetilde{G}(r-k-1)}\left(\mathrm{d}^{1 / 2} \otimes \vartheta_{r-k-1} \otimes \pi^{(k+1)}, \mathrm{d}^{z-3 / 4} \otimes \oplus \vartheta_{r-k-1}\right) \\
& \cong \operatorname{Hom}_{\widetilde{G}(r-k-1)}\left(\vartheta_{r-k-1} \otimes \oplus \vartheta_{r-k-1} \otimes \pi^{(k+1)}, \mathrm{d}^{z-5 / 4}\right),
\end{aligned}
$$

where $\oplus \vartheta_{r-k-1}$ denotes a finite direct sum of exceptional representations formed with respect to various suitable characters. Here we have used (4.7) and Proposition 4.3 from the first line to the second, Proposition 4.2 and Theorem 5.2 from the second to the third, the definitions of $\Psi^{-}, \Psi^{+}$ and the derivative functors from the third to the fourth and (4.7) and Theorem 5.1 from the fourth to the last. All the exceptional representations in (6.6) transform via a suitable character under $\widetilde{Z}_{2}(r-k-1)$. The representation $\pi^{(k+1)}$ is of finite length and comparing the $\widetilde{Z}_{2}(r-k-1)$ characters in both entries of (6.6) we see that the space of homomorphisms is $\{0\}$ provided that no non-zero subquotient of $\pi^{(k+1)}$ has central character, $\alpha$, satisfying $\alpha^{2}=\mathrm{d}^{2 z-5 / 2}$. If this proviso is satisfied then (6.5) shows that $\mathcal{H}_{k}(\pi, z)$ may be regarded as a subspace of the space

$$
\begin{aligned}
& \operatorname{Hom}_{\widetilde{P}(r-k)}\left(\Psi^{+}\left(\vartheta_{r-k-1}\right) \otimes\left(\Phi^{-}\right)^{k}(\pi) \otimes \Phi^{+} \Phi^{-}\left(\vartheta_{r-k}\right), \mathrm{d}^{z}\right) \\
& \cong \operatorname{Hom}_{\widetilde{P}(r-k)}\left(\Psi^{+}\left(\vartheta_{r-k-1}\right) \otimes\left(\Phi^{-}\right)^{k}(\pi), \mathrm{d}^{z-1} \otimes \widehat{\Phi}^{+}\left(\mathrm{d} \otimes \widehat{\Phi^{-}\left(\vartheta_{r-k}\right)}\right)\right) \\
& \left.\cong \operatorname{Hom}_{\widetilde{P}(r-k-1)}\left(\Phi^{-}\left(\Psi^{+}\left(\vartheta_{r-k-1}\right) \otimes\left(\Phi^{-}\right)^{k}(\pi)\right), \mathrm{d}^{z} \otimes \Phi^{-\widehat{\left(\vartheta_{r-k}\right.}}\right)\right) \\
& \cong \operatorname{Hom}_{\widetilde{P}(r-k-1)}\left(\mathrm{d}^{1 / 2} \otimes \vartheta_{r-k-1} \otimes \Phi^{-}\left(\vartheta_{r-k}\right) \otimes\left(\Phi^{-}\right)^{k+1}(\pi), \mathrm{d}^{z}\right) .
\end{aligned}
$$

In this sequence of isomorphisms we have used (4.7) and Proposition 4.3 from line one to line two, Proposition 4.2 from line two to line three, and finally (4.7), the definition of $\Phi^{-}$and the fact that $Y_{r-k}^{*}$ acts trivially on $\Psi^{+}\left(\vartheta_{r-k-1}\right)$ from line three to line four.

Up until now no use has been made of the standing assumption in this section that either $F$ is not dyadic or $r=2$ or 3 . It becomes necessary when we seek to analyze (6.7) further. Applying 
the standard short exact sequence of functors given in Section 3 of [3] to the representation $\Phi^{-}\left(\vartheta_{r-k}\right)$ we obtain a short exact sequence

$$
0 \longrightarrow \Phi^{+}\left(\Phi^{-}\right)^{2}\left(\vartheta_{r-k}\right) \longrightarrow \Phi^{-}\left(\vartheta_{r-k}\right) \longrightarrow \Psi^{+}\left(\vartheta_{r-k}^{(2)}\right) \longrightarrow 0
$$

Since $\vartheta_{r-k}^{(j)}=0$ if $j \geqslant 3$, by Theorem 5.4, we see that all the proper derivatives of the representation $\left(\Phi^{-}\right)^{2}\left(\vartheta_{r-k}\right)$ are zero. But, according to Corollary 5.14 in [2], a non-zero representation must have at least one non-zero proper derivative and we conclude that $\left(\Phi^{-}\right)^{2}\left(\vartheta_{r-k}\right)=0$. Hence (6.8) and Theorem 5.3 give

$$
\Phi^{-}\left(\vartheta_{r-k}\right) \cong \Psi^{+}\left(\vartheta_{r-k}^{(2)}\right) \cong \mathrm{d}^{-1 / 2} \otimes \Psi^{+}\left(\vartheta_{r-k-2}\right)
$$

Using this isomorphism we see that (6.7) is isomorphic to

$$
\operatorname{Hom}_{\widetilde{P}(r-k-1)}\left(\Psi^{+}\left(\vartheta_{r-k-2}\right) \otimes\left(\Phi^{-}\right)^{k+1}(\pi) \otimes \vartheta_{r-k-1}, \mathrm{~d}^{z}\right),
$$

which is, by definition, $\mathcal{H}_{k+1}(\pi, z)$. Thus, if $\pi^{(k+1)}$ has no non-zero subquotient with central character, $\alpha$, satisfying $\alpha^{2}=\mathrm{d}^{2 z-5 / 2}$, then $\mathcal{H}_{k}(\pi, z)$ may be regarded as a subspace of $\mathcal{H}_{k+1}(\pi, z)$.

With the analysis of $\mathcal{H}_{k}(\pi, z)$ complete, it is time to return to the space of $G(r)$-invariant linear functionals whose dimension we are seeking to estimate. In the first part of the proof we saw that this space is isomorphic to (6.3), which is, in turn, isomorphic to $\mathcal{H}_{0}(\pi, 1-s)$. Since we are assuming that $\pi$ is general with respect to $s$, no intermediate derivative of $\pi$ has a non-zero subquotient with central character, $\alpha$, satisfying $\alpha^{2}=\mathrm{d}^{-2 s-1 / 2}$. The second part of the proof shows that, under this condition, $\mathcal{H}_{k}(\pi, 1-s)$ may be regarded as a subspace of $\mathcal{H}_{k+1}(\pi, 1-s)$. Hence $\mathcal{H}_{0}(\pi, 1-s)$ may be regarded as a subspace of

$$
\mathcal{H}_{r-1}(\pi, 1-s)=\operatorname{Hom}_{\widetilde{P}(1)}\left(\Psi^{+}\left(\vartheta_{0}\right) \otimes\left(\Phi^{-}\right)^{r-1}(\pi) \otimes \vartheta_{1}, \mathrm{~d}^{1-s}\right) .
$$

Both $\vartheta_{1}$ and $\Psi^{+}\left(\vartheta_{0}\right)$ are one-dimensional and $\left(\Phi^{-}\right)^{r-1}(\pi)$ is realized on the same space as $\pi^{(r)}$. Hence (6.10) has the same dimension as the space of Whittaker functionals on $\pi$.

COROLlARY 6.1. - Let $\pi$ be a homogeneous admissible representation of $G(r)$ of finite length which is general with respect to $1 / 4$. Then the dimension of $\mathcal{L}(\omega, \nu ; \pi)$ is at most the dimension of the space of Whittaker functionals on $\pi$.

Proof. - Combining parts 5 and 6 of Theorem 5.1, we see that $\vartheta_{r, \nu}$ is isomorphic to a quotient of

$$
i_{(r-1,1),(r)}\left(\mu_{(r),(r-1,1)}^{1 / 4} \otimes \vartheta_{(r-1,1), \nu}\right)
$$

and hence there is an injective map from $\mathcal{L}(\omega, \nu ; \pi)$ into the space of $G(r)$-invariant functionals on

$$
\vartheta_{r, \omega} \otimes i_{(r-1,1),(r)}\left(\mu_{(r),(r-1,1)}^{1 / 4} \otimes \vartheta_{(r-1,1), \nu}\right) \otimes \pi .
$$

The result now follows directly from Theorem 6.1.

Note that if $\pi$ is a cuspidal representation of $G(r)$ then, by Theorem 4.4 of [3], all the intermediate derivatives of $\pi$ are zero and so $\pi$ is automatically general with respect to any $s \in \mathbb{C}$. If $\pi$ is also irreducible then $\pi^{(r)}=1$ and it follows from Corollary 6.1 that the dimension of $\mathcal{L}(\omega, \nu ; \pi)$ is at most one. 
The hypothesis of generality with respect to $1 / 4$ in Corollary 6.1 is necessary. To see this, let us take $\pi$ to be the trivial representation of $G(r)$ with $r \geqslant 2$. Then $\pi$ carries no Whittaker functionals but $\mathcal{L}\left(\omega, \omega^{-1} ; \pi\right)$ is exactly one-dimensional because $\vartheta_{r, \omega}$ is irreducible and its contragredient is isomorphic to $\vartheta_{r, \omega^{-1}}$. The first derivative of $\pi$ is the character $\mathrm{d}^{-1 / 2}$ on $G(r-1)$ and all the higher derivatives are zero. Thus $\pi$ is not general with respect to $1 / 4$.

The following result allows us to study the space $\mathcal{L}(\omega, \nu ; \pi)$ inductively when $\pi$ is parabolically induced from a smaller group.

THEOREM 6.2. - Let $\rho$ be a homogeneous admissible representation of $G(r-1)$ of finite length. If $r$ is odd then define a character $\alpha$ of $F^{\times}$by

$$
\alpha(z)=\omega_{\rho}\left(z I_{r-1}\right)^{-1} \cdot(\omega \cdot \nu)\left(z I_{r}\right)
$$

where $\omega_{\rho}$ is the central character of $\rho$. If $r$ is even, then define $\alpha$ by (6.11) for $z \in\left(F^{\times}\right)^{2}$ and extend it in any way to a character of $F^{\times}$. Let $\pi=i_{(r-1, r),(r)}(\rho \otimes \alpha)$. If $r$ is odd, then there is an exact sequence

$$
\{0\} \longrightarrow \mathcal{L}(\eta ; \rho) \longrightarrow \mathcal{L}(\omega, \nu ; \pi) \longrightarrow \mathcal{L}\left(\omega^{\prime}, \nu^{\prime} ; \rho^{(1)}\right)
$$

where $\eta$ is the unique suitable character of $Z(\widetilde{G}(r-1))$ and $\omega^{\prime}$ and $\nu^{\prime}$ are suitable characters of $Z(\widetilde{G}(r-2))$ related to $\omega$ and $\nu$ by the equations

$$
\omega=\left.\left(\omega^{\prime} \otimes\left(\chi_{0}\right)_{\psi}\right)\right|_{Z(\widetilde{G}(r))} \quad \text { and } \quad \nu=\left.\left(\nu^{\prime} \otimes\left(\chi_{0}\right)_{\psi}\right)\right|_{Z(\widetilde{G}(r))} .
$$

If $r$ is even, then $\omega=\nu$ and there is a space $V$ which completes the diagram

$$
\{0\} \longrightarrow \bigoplus_{\eta_{1}, \eta_{2}} \mathcal{L}\left(\eta_{1}, \eta_{2} ; \rho\right) \longrightarrow \stackrel{\mathcal{L}(\omega ; \pi)}{\longrightarrow} \longrightarrow \mathcal{L}\left(\omega^{\prime} ; \rho^{(1)}\right)
$$

with the row exact. Here $\omega^{\prime}$ is the unique suitable character on $Z(\widetilde{G}(r-2))$ and the direct sum is over all pairs of suitable characters $\eta_{1}$ and $\eta_{2}$ of $Z(\widetilde{G}(r-1))$. The space $V$ is independent of the extension of $\alpha$ from $\left(F^{\times}\right)^{2}$ to $F^{\times}$and if $V$ is non-zero, then $\mathcal{L}(\omega ; \pi)$ is non-zero for at least one choice of this extension.

Proof. - The space $\mathcal{L}(\omega, \nu ; \pi)$ is isomorphic to

$$
\begin{aligned}
& \operatorname{Hom}_{G(r)}\left(\vartheta_{r, \omega} \otimes \vartheta_{r, \nu} \otimes \pi, 1\right) \\
& \quad \cong \operatorname{Hom}_{G(r)}\left(\vartheta_{r, \omega} \otimes \vartheta_{r, \nu}, i_{(r-1,1),(r)}\left(\widehat{\rho} \otimes \alpha^{-1}\right)\right) \\
& \cong \operatorname{Hom}_{Q((r-1,1))}\left(\vartheta_{r, \omega} \otimes \vartheta_{r, \nu}, \Psi^{+}\left(\widehat{\rho} \otimes \alpha^{-1}\right)\right) .
\end{aligned}
$$

We note explicitly that the symbol $\hat{\rho}$ in (6.13) refers to the contragredient of $\rho$ as a representation of $G(r-1)$. The characters of the representations in the first and second places in (6.13) under $p_{r}(Z(\widetilde{G}(r)))$ have been arranged to be equal. If $r$ is odd then, since $Q((r-1,1))=$ $p_{r}(Z(\widetilde{G}(r))) \cdot P(r)$, it follows that (6.13) is isomorphic to

$$
\operatorname{Hom}_{P(r)}\left(\vartheta_{r, \omega} \otimes \vartheta_{r, \nu}, \Psi^{+}(\widehat{\rho})\right)
$$


If $r$ is even then (6.14) will be the space denoted by $V$ in the statement. It evidently does not depend on the extension of $\alpha$ which was chosen and since the $P(r)$-intertwining property required of elements of $(6.14)$ is less restrictive than the $Q((r-1,1))$-intertwining property required of elements of $(6.13), \mathcal{L}(\omega ; \pi)$ is always a subspace of $(6.14)$. Since $Z(r) / Z_{2}(r)$ is a finite abelian group, any element of (6.14) may be expressed as a sum of linear maps between the underlying spaces each of which is $P(r)$-intertwining and transforms under $Z(r)$ by one of the square-trivial characters. These summands are elements of the various $\mathcal{L}(\omega ; \pi)$ where $\pi$ is formed with the various possible extensions of $\alpha$ to $F^{\times}$. If the original map is non-zero, then at least one of its summands must be non-zero and this shows that if $V \neq\{0\}$ then $\mathcal{L}(\omega ; \pi) \neq\{0\}$ for at least one choice of extension.

After these observations it remains to analyze (6.14). We shall begin with the short exact sequence

$$
0 \longrightarrow \Phi^{+} \Phi^{-}\left(\vartheta_{r, \omega}\right) \otimes \vartheta_{r, \nu} \longrightarrow \vartheta_{r, \omega} \otimes \vartheta_{r, \nu} \longrightarrow \Psi^{+} \Psi^{-}\left(\vartheta_{r, \omega}\right) \otimes \vartheta_{r, \nu} \longrightarrow 0
$$

which yields an exact sequence

$$
\begin{aligned}
\{0\} & \longrightarrow \operatorname{Hom}_{P(r)}\left(\Psi^{+} \Psi^{-}\left(\vartheta_{r, \omega}\right) \otimes \vartheta_{r, \nu}, \Psi^{+}(\widehat{\rho})\right) \\
& \longrightarrow \operatorname{Hom}_{P(r)}\left(\vartheta_{r, \omega} \otimes \vartheta_{r, \nu}, \Psi^{+}(\widehat{\rho})\right) \\
& \longrightarrow \operatorname{Hom}_{P(r)}\left(\Phi^{+} \Phi^{-}\left(\vartheta_{r, \omega}\right) \otimes \vartheta_{r, \nu}, \Psi^{+}(\widehat{\rho})\right) .
\end{aligned}
$$

The first Hom-space in (6.15) is easy to analyze. Indeed, by Proposition 4.2, statement 1, it is isomorphic to

$$
\operatorname{Hom}_{G(r-1)}\left(\mathrm{d}^{1 / 2} \otimes \Psi^{-}\left(\vartheta_{r, \omega}\right) \otimes \Psi^{-}\left(\vartheta_{r, \nu}\right), \widehat{\rho}\right) \cong \operatorname{Hom}_{G(r-1)}\left(\mathrm{d}^{1 / 2} \otimes \vartheta_{r, \omega}^{(1)} \otimes \vartheta_{r, \nu}^{(1)} \otimes \rho, 1\right)
$$

and, by Theorem 5.2, this is isomorphic to $\mathcal{L}(\eta ; \rho)$ if $r$ is odd and to $\bigoplus_{\eta_{1}, \eta_{2}} \mathcal{L}\left(\eta_{1}, \eta_{2} ; \rho\right)$ if $r$ is even.

We now turn to analyze the third Hom-space in (6.15). First, we have

$$
\begin{aligned}
& \operatorname{Hom}_{P(r)}\left(\Phi^{+} \Phi^{-}\left(\vartheta_{r, \omega}\right) \otimes \vartheta_{r, \nu}, \Psi^{+}(\widehat{\rho})\right) \\
& \cong \operatorname{Hom}_{P(r)}\left(\Phi^{+} \Phi^{-}\left(\vartheta_{r, \omega}\right) \otimes \vartheta_{r, \nu}, \mathrm{d} \otimes \widehat{\Psi^{+}(\rho)}\right) \\
& \left.\cong \operatorname{Hom}_{\widetilde{P}(r)}\left(\mathrm{d}^{-1} \otimes \Psi^{+}(\rho) \otimes \vartheta_{r, \nu}, \Phi^{+} \widehat{\Phi^{-}\left(\vartheta_{r, \omega}\right.}\right)\right)
\end{aligned}
$$

by Proposition 4.3 and (4.7). This, in turn, is isomorphic to

$$
\begin{aligned}
& \operatorname{Hom}_{\widetilde{P}(r)}\left(\mathrm{d}^{-1} \otimes \Psi^{+}(\rho) \otimes \vartheta_{r, \nu}, \mathrm{d}^{-1} \otimes \widehat{\Phi}^{+}\left(\mathrm{d} \otimes \widehat{\Phi^{-\left(\vartheta_{r, \omega}\right.}}\right)\right) \\
& \left.\cong \operatorname{Hom}_{\widetilde{P}(r-1)}\left(\mathrm{d}^{1 / 2} \otimes \rho \otimes \Phi^{-}\left(\vartheta_{r, \nu}\right), \mathrm{d} \otimes \widehat{\Phi^{-\left(\vartheta_{r, \omega}\right.}}\right)\right) \\
& \cong \operatorname{Hom}_{P(r-1)}\left(\mathrm{d}^{-1 / 2} \otimes \rho \otimes \Phi^{-}\left(\vartheta_{r, \omega}\right) \otimes \Phi^{-}\left(\vartheta_{r, \nu}\right), 1\right)
\end{aligned}
$$

where we have used Proposition 4.3 to reach the first line, Proposition 4.2 from the first to the second and then (4.7) to reach the last line. From (6.9) and Theorem 5.3, we know that

$$
\Phi^{-}\left(\vartheta_{r, \omega}\right) \cong \Psi^{+}\left(\vartheta_{r, \omega}^{(2)}\right) \cong \mathrm{d}^{-1 / 2} \otimes \Psi^{+}\left(\vartheta_{r-2, \omega^{\prime}}\right),
$$

where $\omega^{\prime}$ is as described in the statement, and similarly with $\omega$ replaced by $\nu$. Hence (6.16) is isomorphic to 


$$
\begin{aligned}
& \operatorname{Hom}_{P(r-1)}\left(\mathrm{d}^{-3 / 2} \otimes \rho \otimes \Psi^{+}\left(\vartheta_{r-2, \omega^{\prime}}\right) \otimes \Psi^{+}\left(\vartheta_{r-2, \nu^{\prime}}\right), 1\right) \\
& \quad \cong \operatorname{Hom}_{P(r-1)}\left(\mathrm{d}^{-3 / 2} \otimes \Psi^{+}\left(\vartheta_{r-2, \omega^{\prime}}\right) \otimes \Psi^{+}\left(\vartheta_{r-2, \nu^{\prime}}\right), \widehat{\rho}\right) \\
& \cong \operatorname{Hom}_{P(r-1)}\left(\mathrm{d}^{-1} \otimes \Psi^{+}\left(\vartheta_{r-2, \omega^{\prime}} \otimes \vartheta_{r-2, \nu^{\prime}}\right), \widehat{\rho}\right),
\end{aligned}
$$

where the symbol $\hat{\rho}$ now denotes the contragredient of $\left.\rho\right|_{P(r-1)}$. Finally, this is isomorphic to

$$
\begin{aligned}
& \operatorname{Hom}_{G(r-2)}\left(\mathrm{d}^{-1} \otimes \vartheta_{r-2, \omega^{\prime}} \otimes \vartheta_{r-2, \nu^{\prime}}, \widehat{\Psi}^{-}(\widehat{\rho})\right) \\
& \cong \operatorname{Hom}_{G(r-2)}\left(\mathrm{d}^{-1} \otimes \vartheta_{r-2, \omega^{\prime}} \otimes \vartheta_{r-2, \nu^{\prime}}, \mathrm{d}^{-1} \otimes \widehat{\Psi^{-}(\rho)}\right) \\
& \cong \operatorname{Hom}_{G(r-2)}\left(\vartheta_{r-2, \omega^{\prime}} \otimes \vartheta_{r-2, \nu^{\prime}} \otimes \Psi^{-}(\rho), 1\right) \\
& \quad=\mathcal{L}\left(\omega^{\prime}, \nu^{\prime} ; \rho^{(1)}\right),
\end{aligned}
$$

where we have used Proposition 4.2 to reach the first line, Proposition 4.3 from the first line to the second and (4.7) from the second to the third.

Our first use for Theorem 6.2 will be as an aid in understanding $\mathcal{L}(\omega, \nu ; \pi)$ when $\pi$ is a representation of the principal series. For notational simplicity, we shall let $\chi=\left(\chi_{1}, \ldots, \chi_{r}\right)$ be a character of $G\left(\gamma_{0}\right)$ and write $\mathbb{I}(\chi)=i_{\gamma_{0},(r)}\left(\chi_{1} \otimes \cdots \otimes \chi_{r}\right)$ for the principal series representation of $G(r)$ arising by normalized parabolic induction from $\chi$.

DEFINITION 6.2. - A character $\chi=\left(\chi_{1}, \ldots, \chi_{r}\right)$ of $G\left(\gamma_{0}\right)$ will be called balanced if there is an involution $j \mapsto j^{*}$ of the set $\{1, \ldots, r\}$ such that, for all $1 \leqslant j \leqslant r, \chi_{j}^{2} \chi_{j^{*}}^{2}=1$ if $j \neq j^{*}$ and $\chi_{j}^{2}=1$ if $j=j^{*}$.

\section{THEOREM 6.3. - If $\mathcal{L}(\omega, \nu ; \mathbb{I}(\chi)) \neq\{0\}$ for some choice of $\omega$ and $\nu$, then $\chi$ is balanced.}

Proof. - We note that, if $r=1$ or 2 , then $\chi$ is balanced if and only if $\mathbb{I}(\chi)$ has square-trivial central character. Since $\vartheta_{r, \omega} \otimes \vartheta_{r, \nu}$ transforms under $p_{r}(Z(\widetilde{G}(r)))$ by $\omega \cdot \nu$, a necessary condition for $\mathcal{L}(\omega, \nu ; \pi) \neq\{0\}$ is that $\omega_{\pi}=\omega \cdot \nu$ on $p_{r}(Z(\widetilde{G}(r)))$. In particular, $\omega_{\pi}$ must be square-trivial. It follows that the claim is true when $r \leqslant 2$.

We shall proceed by induction on $r$. Suppose that $r \geqslant 3$ and that $\mathcal{L}(\omega, \nu ; \mathbb{I}(\chi)) \neq\{0\}$. By the transitivity of induction we have

$$
\mathbb{I}(\chi) \cong \operatorname{ind}_{Q((r-1,1))}^{G(r)}\left(\mathbb{I}\left(\chi_{1}, \ldots, \chi_{r-1}\right) \otimes \chi_{r}\right)
$$

If we set $\rho=\mathbb{I}\left(\chi_{1}, \ldots, \chi_{r-1}\right)$, then $\omega_{\rho}\left(z I_{r-1}\right)=\prod_{j=1}^{r-1} \chi_{j}(z)$ and it follows from the remarks in the first paragraph that $\omega_{\rho}\left(z I_{r-1}\right) \cdot \chi_{r}(z)=(\omega \cdot \nu)\left(z I_{r}\right)$ for $z I_{r} \in p_{r}(Z(\widetilde{G}(r)))$. Thus, regardless of the parity of $r, \alpha=\chi_{r}$ is one appropriate choice to use in applying Theorem 6.2. We conclude from that theorem that either $\mathcal{L}\left(\eta_{1}, \eta_{2} ; \rho\right) \neq\{0\}$ for some choice of suitable characters $\eta_{1}$ and $\eta_{2}$ or that $\mathcal{L}\left(\omega^{\prime}, \nu^{\prime} ; \rho^{(1)}\right) \neq\{0\}$. If the first possibility obtains then we conclude inductively that $\left(\chi_{1}, \ldots, \chi_{r-1}\right)$ is balanced. In particular, $\prod_{j=1}^{r-1} \chi_{j}^{2}=1$ and hence $\chi_{r}^{2}=1$. If we extend the involution $j \mapsto j^{*}$ of $\{1, \ldots, r-1\}$ to $\{1, \ldots, r\}$ by setting $r^{*}=r$ then we obtain an involution which shows that $\chi$ is balanced. This completes the induction step in this case.

Suppose now that the second of the above possibilities obtains. Using Corollary 4.6 of [3] (the so-called Leibniz rule for derivatives) we see that $\rho^{(1)}$ is glued from the representations $\rho_{\ell}=$ $\mathbb{I}\left(\chi_{1}, \ldots, \widehat{\chi_{\ell}}, \ldots, \chi_{r-1}\right)$, where the hat denotes omission. Since $\mathcal{L}\left(\omega^{\prime}, \nu^{\prime} ; \rho^{(1)}\right) \neq\{0\}$, it follows that we must have $\mathcal{L}\left(\omega^{\prime}, \nu^{\prime} ; \rho_{\ell}\right) \neq\{0\}$ for some $\ell$. Then, by induction, $\left(\chi_{1}, \ldots, \widehat{\chi_{\ell}}, \ldots, \chi_{r-1}\right)$ is balanced for that value of $\ell$. In particular, $\prod_{j=1, j \neq \ell}^{r-1} \chi_{j}^{2}=1$ and, since $\prod_{j=1}^{r} \chi_{j}^{2}=1$, it follows that $\chi_{\ell}^{2} \chi_{r}^{2}=1$. Thus, if we take the involution $j \mapsto j^{*}$ of $\{1, \ldots, r\} \backslash\{\ell, r\}$ corresponding to the 
induction datum of $\rho_{\ell}$ being balanced and extend it to $\{1, \ldots, r\}$ by setting $\ell^{*}=r$ then we have an involution showing that $\chi$ is balanced. This completes the induction step in this case.

It is not too hard to show that, if $\chi_{1}, \ldots, \chi_{r}$ are square-trivial characters satisfying $\prod_{j=1}^{r} \chi_{j}=$ $\omega \cdot \nu$, then $\mathcal{L}(\omega, \nu ; \mathbb{I}(\chi)) \neq\{0\}$. Thus, in the definition of balanced, $\chi_{j}^{2}$ cannot be replaced by $\chi_{j}$ if Theorem 6.3 is to be true.

The next result gives information on the dimension of the space $\mathcal{L}(\omega, \nu ; \mathbb{I}(\chi))$. It overlaps with Theorem 6.1, but does not have exactly the same range of applicability, and the technique is different. As usual, we call $\chi$ regular if it is not fixed by any non-identity element of the Weyl group; here this simply means that the characters $\chi_{1}, \ldots, \chi_{r}$ are distinct.

THEOREM 6.4. - Suppose that $\chi=\left(\chi_{1}, \ldots, \chi_{r}\right)$ is balanced and that $\chi^{2}$ is regular. Then, for any $\omega$ and $\nu$,

$$
\operatorname{dim}_{\mathbb{C}}(\mathcal{L}(\omega, \nu ; \mathbb{I}(\chi))) \leqslant 1
$$

Proof. - We shall use induction on $r$, beginning with the cases where $r=1$ and $r=2$. If $r=1$ then $\mathbb{I}(\chi)=\chi_{1}$ is a square-trivial character and so the dimension of $\mathcal{L}(\omega, \nu ; \mathbb{I}(\chi))$ is one if $\chi_{1}=\omega \cdot \nu$ and zero otherwise. Thus (6.17) holds in this case. If $r=2$ then we have an induction datum $\chi=\left(\chi_{1}, \chi_{2}\right)$ which satisfies $\chi_{1}^{2} \chi_{2}^{2}=1$ and $\chi_{1}^{2} \neq \chi_{2}^{2}$; in particular, $\chi_{1}^{2} \neq 1$. Thus $\mathcal{L}\left(\eta_{1}, \eta_{2} ; \chi_{1}\right)=\{0\}$ for all suitable characters $\eta_{1}$ and $\eta_{2}$. Using Theorem 6.2 with $\rho=\chi_{1}$ and $\alpha=\chi_{2}$ we obtain an injection $\mathcal{L}(\omega ; \mathbb{I}(\chi)) \hookrightarrow \mathcal{L}\left(\omega^{\prime} ; \chi_{1}^{(1)}\right)$. But $\chi_{1}^{(1)}$ is the trivial representation of $G(0)$ and hence $\mathcal{L}\left(\omega^{\prime} ; \chi_{1}^{(1)}\right) \cong \mathbb{C}$. Thus (6.17) holds in this case also.

Now suppose that $r \geqslant 3$. We shall apply Theorem 6.2 with $\rho=\mathbb{I}\left(\chi_{1}, \ldots, \chi_{r-1}\right)$ and $\alpha=\chi_{r}$. First, assume that $\chi_{r}^{2} \neq 1$. Then $\omega_{\rho}^{2}=\chi_{r}^{-2} \neq 1$ and so $\mathcal{L}\left(\eta_{1}, \eta_{2} ; \rho\right)=\{0\}$ for all suitable characters $\eta_{1}$ and $\eta_{2}$. Theorem 6.2 then implies that there is an injection

$$
\mathcal{L}(\omega, \nu ; \mathbb{I}(\chi)) \hookrightarrow \mathcal{L}\left(\omega^{\prime}, \nu^{\prime} ; \rho^{(1)}\right)
$$

As in the proof of Theorem $6.3, \rho^{(1)}$ is glued from the representations $\rho_{\ell}=\mathbb{I}\left(\chi_{1}, \ldots, \widehat{\chi_{\ell}}, \ldots\right.$, $\left.\chi_{r-1}\right)$ for $\ell=1, \ldots,(r-1)$. If $\ell \neq \ell^{\prime}$ but the central characters of $\rho_{\ell}$ and $\rho_{\ell^{\prime}}$ have equal squares, then we would conclude that $\chi_{\ell}^{2}=\chi_{\ell^{\prime}}^{2}$, contradicting the regularity assumption. Thus the squares of the central characters of the $\rho_{\ell}$ are all distinct. It follows that $\rho^{(1)} \cong \bigoplus_{\ell=1}^{r-1} \rho_{\ell}$ and that at most one of the $\rho_{\ell}$ has square-trivial central character.

Suppose that $\rho_{k}$ does have square-trivial central character; we claim that its induction datum is balanced and that the square of its induction datum is regular. The regularity is clear. On the other hand, we have $\prod_{\ell=1, \ell \neq k}^{r-1} \chi_{j}^{2}=1$ and hence $\chi_{k}^{2} \chi_{r}^{2}=1$. Let $j \mapsto j^{*}$ be an involution which corresponds to $\chi$ being balanced. Since $\chi_{k}^{2} \neq 1$ and $\chi_{r}^{2} \neq 1, k^{*} \neq k$ and $r^{*} \neq r$. Thus we may define a new involution $j \mapsto j^{\prime}$ by $j^{\prime}=j^{*}$ if $j \notin\left\{k, k^{*}, r, r^{*}\right\}, k^{\prime}=r$ and $\left(k^{*}\right)^{\prime}=r^{*}$ and $j \mapsto j^{\prime}$ will be a second involution showing that $\chi$ is balanced (of course, $j \mapsto j^{*}$ and $j \mapsto j^{\prime}$ might be equal). Restricting $j \mapsto j^{\prime}$ to the set $\{1, \ldots, r\} \backslash\{k, r\}$ gives an involution which shows that the character $\left(\chi_{1}, \ldots, \widehat{\chi_{k}}, \ldots, \chi_{r-1}\right)$ is balanced. Our claim follows.

From the argument of the previous two paragraphs we conclude that

$$
\mathcal{L}\left(\omega^{\prime}, \nu^{\prime} ; \rho^{(1)}\right) \cong \bigoplus_{\ell=1}^{r-1} \mathcal{L}\left(\omega^{\prime}, \nu^{\prime} ; \rho_{\ell}\right),
$$

that all but one of the summands on the right of (6.19) are zero (on central character grounds) and that if one of the summands is non-zero then the induction datum of the corresponding $\rho_{\ell}$ 
is balanced and its square is regular. Thus if one of the summands is non-zero it is at most one-dimensional by the induction hypothesis. Hence the left-hand side of (6.19) is at most one-dimensional and it follows from $(6.18)$ that $\mathcal{L}(\omega, \nu ; \mathbb{I}(\chi))$ is at most one-dimensional. This completes the induction in this case.

Now, assume that $\chi_{r}^{2}=1$. Then $\chi_{j}^{2} \neq 1$ for all $j=1, \ldots,(r-1)$ and so the involution $j \mapsto j^{*}$ of $\{1, \ldots, r\}$ which corresponds to $\chi$ being balanced must satisfy $r^{*}=r$. If $r$ were even then the restriction of $j \mapsto j^{*}$ to $\{1, \ldots, r-1\}$ would necessarily have a fixed point and this would give us some $j$ between 1 and $(r-1)$ such that $\chi_{j}^{2}=1$. This is impossible and so $r$ must be odd. Hence we have an exact sequence

$$
\{0\} \longrightarrow \mathcal{L}(\eta, \rho) \longrightarrow \mathcal{L}(\omega, \nu ; \mathbb{I}(\chi)) \longrightarrow \mathcal{L}\left(\omega^{\prime}, \nu^{\prime} ; \rho^{(1)}\right)
$$

from Theorem 6.2. The central character of $\rho$ is square-trivial and thus if $\rho_{\ell}$, as above, had square-trivial central character for some $\ell$ then we would conclude that $\chi_{\ell}^{2}=1$, thus contradicting regularity. Therefore no $\rho_{\ell}$ has square-trivial central character, $\mathcal{L}\left(\omega^{\prime}, \nu^{\prime} ; \rho_{\ell}\right)=\{0\}$ for all $\ell$ and hence $\mathcal{L}\left(\omega^{\prime}, \nu^{\prime} ; \rho^{(1)}\right)=\{0\}$. From this and (6.20) we obtain an isomorphism $\mathcal{L}(\omega, \nu ; \mathbb{I}(\chi)) \cong$ $\mathcal{L}(\eta ; \rho)$. Since we have seen that $r^{*}=r$, the induction datum of $\rho$ is balanced and its square is clearly regular. Thus $\mathcal{L}(\eta ; \rho)$ is at most one-dimensional, by the induction hypothesis, and consequently $\mathcal{L}(\omega, \nu ; \mathbb{I}(\chi))$ is at most one-dimensional. This completes the inductive step in this case and hence the proof.

THEOREM 6.5. - If $\pi$ is an irreducible admissible representation of $G(2)$, then $\operatorname{dim}_{\mathbb{C}} \mathcal{L}(\omega ; \pi)$ $\leqslant 1$. If $\pi$ is an irreducible admissible representation of $G(3)$, then $\operatorname{dim}_{\mathbb{C}} \mathcal{L}(\omega, \nu ; \pi) \leqslant 1$.

Proof. - If $\pi$ is a cuspidal representation of $G(2)$, then, as we have remarked previously, $\pi$ is general with respect to $1 / 4$ and so Corollary 6.1 gives the claim, since cuspidal representations have a unique Whittaker model. Otherwise, $\pi$ is a constituent of some principal series representation $\mathbb{I}(\chi)$. Replacing $\chi=\left(\chi_{1}, \chi_{2}\right)$ by $\left(\chi_{2}, \chi_{1}\right)$, if necessary, we may assume that $\pi$ occurs as a quotient of $\mathbb{I}(\chi)$. Then $\mathcal{L}(\omega ; \pi)$ is a subspace of $\mathcal{L}(\omega ; \mathbb{I}(\chi))$ and so it suffices to show that this last space is at most one-dimensional for all $\chi$.

If $\mathcal{L}(\omega ; \mathbb{I}(\chi)) \neq\{0\}$ then $\chi_{1}^{2} \chi_{2}^{2}=1$. The required result follows from Theorem 6.4 unless $\chi^{2}$ is irregular; that is, unless $\chi_{1}^{2}=\chi_{2}^{2}$. Let us suppose that this is so. The only intermediate derivative of $\mathbb{I}(\chi)$ is $\mathbb{I}(\chi)^{(1)}$, which is glued from $\chi_{1}$ and $\chi_{2}$. We cannot have $\chi_{j}^{2}=|\cdot|^{-1}$ for $j=1$ or 2 since $\chi_{j}^{4}=1$ and so $\mathbb{I}(\chi)$ is general with respect to $1 / 4$. By Corollary 6.1 , the dimension of $\mathcal{L}(\omega ; \mathbb{I}(\chi))$ is at most the dimension of the space of Whittaker models on $\mathbb{I}(\chi)$ and it is well known that this is one.

We now consider the situation on $G(3)$. First, observe that it suffices to demonstrate the claim when $\nu=\omega^{-1}$ since twisting both $\pi$ and the second exceptional representation by a squaretrivial character reduces the general case to this. If $\pi$ is cuspidal then the claim follows from Corollary 6.1 as before. Next suppose that $\pi$ is a constituent of $i_{(2,1),(3)}(\rho \otimes \alpha)$, where $\rho$ is a cuspidal representation of $G(2)$. According to Theorem 4.2 of [3], this induced representation is irreducible and hence equal to $\pi$. By Theorem 6.2 we have an exact sequence

$$
\{0\} \longrightarrow \mathcal{L}(\eta ; \rho) \longrightarrow \mathcal{L}\left(\omega, \omega^{-1} ; \pi\right) \longrightarrow \mathcal{L}\left(\omega^{\prime},\left(\omega^{\prime}\right)^{-1} ; \rho^{(1)}\right)
$$

and since $\rho^{(1)}=0$ it follows that $\mathcal{L}\left(\omega, \omega^{-1} ; \pi\right)$ is isomorphic to $\mathcal{L}(\eta ; \rho)$. From the first part of the proof, this space is at most one-dimensional and we obtain the required result for these $\pi$. Finally, $\pi$ might be a constituent of some principal series representation $\mathbb{I}(\chi)$. In this case our claim is the main result of [15]. This completes the proof. 


\title{
Acknowledgements
}

This work constitutes a part of my thesis, written at Oklahoma State University under the direction of Dr James Cogdell. I would like to thank him for his guidance. I am also grateful to the mathematics department at OSU for its financial and administrative support and for providing a comfortable and stimulating atmosphere for research.

\section{REFERENCES}

[1] Banks W.D., LeVy J., SePanski M.R., Block-compatible metaplectic cocycles, J. Reine Angew. Math. 507 (1999) 131-163.

[2] Bernstein J.N., Zelevinsky A.V., Representations of the group GL $(n, F)$ where $F$ is a nonarchimedean local field, Usp. Mat. Nauk. 31 (1976) 5-70.

[3] BeRnStein J.N., ZelEVINSKY A.V., Induced representations of reductive p-adic groups I, Ann. Scient. École Norm. Sup. 10 (1977) 441-472.

[4] Borel A., Linear Algebraic Groups, 2nd enlarged edition, Graduate Texts in Math., Vol. 126, Springer-Verlag, New York, 1991.

[5] Bump D., GinZbUrg D., Symmetric square L-functions on GL (r), Ann. of Math. 136 (1992) 137205.

[6] Flicker Y.Z., KaZHDAn D.A., Metaplectic correspondence, Publ. Math. Inst. Hautes Études Sci. 64 (1989) 53-110.

[7] Flicker Y.Z., KAZHDAN D.A., SAVIN G., Explicit realization of a metaplectic representation, J. d'Anal. Math. 55 (1990) 17-39.

[8] Gelbart S., Piatetski-Shapiro I.I., Distinguished representations and modular forms of halfintegral weight, Invent. Math. 59 (1980) 145-188.

[9] Gelfand I.M., KaZhDan D.A., Representations of GL $(n, K)$, in: Lie Groups and Their Representations 2, Akadèmiai Kiado, Budapest, 1974.

[10] HUANG J.-S., The unitary dual of the universal covering group of GL $(n, \mathbb{R})$, Duke Math. J. 61 (1990) 705-745.

[11] Kable A.C., The main involutions of the metaplectic group, Proc. Amer. Math. Soc. 127 (1999) 955-962.

[12] KaZhdan D.A., Patterson S.J., Metaplectic forms, Publ. Math. Inst. Hautes Études Sci. 59 (1984) $35-142$.

[13] Matsumoto H., Sur les sous-groupes arithmétiques des groupes semi-simples déployés, Ann. Scient. École Norm. Sup. 2 (1969) 1-62.

[14] Pras AD D., Some applications of seesaw duality to branching laws, Math. Ann. 304 (1996) 1-20.

[15] S AVIN G., On the tensor product of theta representations of GL (3), Pacific J. Math. 154 (1992) 369380.

[16] WeIl A., Sur certains groupes d'opérateurs unitaires, Acta Math. 111 (1964) 143-211.

[17] WeIl A., Basic Number Theory, 3rd edition, Grund. Math. Wiss., Vol. 144, Springer-Verlag, New York, 1974.

\author{
Anthony C. KABLE \\ Department of Mathematics, \\ Malott Hall, \\ Cornell University, \\ Ithaca, NY 14853-4201, USA \\ Current address: \\ Department of Mathematics, \\ Oklahoma State University, \\ Stillwater, OK 74078, USA \\ E-mail: akable@math.okstate.edu
}

